\title{
Nonlinear dynamics of a new seasonal epidemiological model with age-structure and nonlinear incidence rate
}

\author{
Abraham J. Arenas ${ }^{1} \cdot$ Gilberto González-Parra ${ }^{2}$ (D) - Nicolás De La Espriella ${ }^{3}$
}

Received: 25 November 2020 / Revised: 18 January 2021 / Accepted: 20 January 2021 /

Published online: 11 February 2021

(c) SBMAC - Sociedade Brasileira de Matemática Aplicada e Computacional 2021

\begin{abstract}
In this article, we study the dynamics of a new proposed age-structured population mathematical model driven by a seasonal forcing function that takes into account the variability of the climate. We introduce a generalized force of infection function to study different potential disease outcomes. Using nonlinear analysis tools and differential inequalities theorems, we obtain sufficient conditions that guarantee the existence of a positive periodic solution. Moreover, we provide sufficient conditions that assure the global attractivity of the positive periodic solution. Numerical results corroborate the theoretical results in the sense that the solutions are positive and the periodic solution is a global attractor. This type of models are important, since they take into account the variability of the weather and the impact on some epidemics such as the current COVID-19 pandemic.
\end{abstract}

Keywords Seasonal epidemic model $\cdot$ Age-structured $\cdot$ Mathematical modeling $\cdot$ Global attraction $\cdot$ Positive periodic solution

Mathematics Subject Classification $34 \mathrm{C} 25 \cdot 34 \mathrm{C} 60 \cdot 37 \mathrm{~N} 25$

\section{Introduction}

Mathematical modeling of dynamical systems is a useful tool to analyze a variety of infectious diseases, to understand their dynamical behavior, to predict their impact on different populations, and to study how different factors can impact the evolution of diseases. One

Communicated by Valeria Neves Domingos Cavalcanti.

$凶$ Gilberto González-Parra

Gilberto.GonzalezParra@nmt.edu

Abraham J. Arenas

aarenas@correo.unicordoba.edu.co

Nicolás De La Espriella

ndelaespriella@correo.unicordoba.edu.co

1 Departamento de Matemáticas y Estadística, Universidad de Córdoba, Montería, Colombia

2 Department of Mathematics, New Mexico Tech, Socorro, NM, USA

3 Departamento de Física y Electrónica, Universidad de Córdoba, Montería, Colombia 
preferred technique to model the behavior of communicable diseases in the population is using systems of ordinary differential equations, where the state variables represent different sub-populations. These systems could be autonomous or nonautonomous depending on the nature of the parameters of the equations. For some infectious diseases and for some particular situations, it makes sense to consider parameters varying over the time, and then, a nonautonomous system is obtained. For instance, there are many diseases that vary depending on the environment conditions. Thus, if we take into account that in many regions around the world, there are four seasons, it seems logical to include the variability of the environment (Shobugawa et al. 2017; Vega et al. 2017; Paiva et al. 2012; Ferrero et al. 2016; Noyola and Mandeville 2008; Traoré and Sangaré 2017; Corberán-Vallet et al. 2018). Moreover, several researchers have remarked and studied the effects of climate change on diseases and ecology (Donaldson 2006; Fagan et al. 2014; Sengupta and Das 2019; Sekerci and Petrovskii 2015; Hurtado et al. 2014; Okuneye et al. 2018; Posny and Wang 2014; Codeço 2001). Then, one approach to include the effect of the weather in the aforementioned mathematical models is to allow time varying parameters. For instance, one disease that shows seasonal behavior is the respiratory syncytial virus (RSV) (Shobugawa et al. 2017; Vega et al. 2017; Paiva et al. 2012; Ferrero et al. 2016; Noyola and Mandeville 2008; Arenas et al. 2009; Jornet-Sanz et al. 2017).

In this paper, we study the dynamics of a developed age-structured population mathematical model driven by a seasonal forcing function to take into account the variability of the climate. In particular, the proposed model is an SEIR (susceptible-exposed-infectedrecovered) type, which is suitable to model the dynamics of several diseases such as influenza, cholera, dengue, RSV, and others (Brauer 2017; Hethcote and van den Driessche 1991). For instance in Hogan et al. (2016b), the authors used an SEIRS model, to explore how the birth rate, varying immunity and seasonality, influences the different dynamics of RSV. Moreover, some authors have pointed out the relation between weather and the current COVID-19 pandemic (Azuma et al. 2020; Bashir et al. 2020; Malki et al. 2020).

Another important aspect of using this type of mathematical models is that once time the model is constructed, it is possible to fit the mathematical model to real data related to infected individuals to estimate the values of the unknown parameters to study different future outcomes or to study the impact of some health policies. It has been argued that nonautonomous models are in some cases more suitable, since the coefficients of the system are varying with the time; for example, the coefficients could be changed with the seasonal factors (White et al. 2007; Arenas et al. 2009; Rogovchenko and Rogovchenko 2009; Rosa and Torres 2018; González-Parra et al. 2009; Bezruchko and Smirnov 2000).

After Kermack-McKendrick published a deterministic model to represent disease transmission, many papers have been published in the direction of generalizing the rate of infection. In Capasso and Serio (1978), a generalization of the Kermack model was proposed, introducing an interaction term in which the dependence on the number of infectious occurs through a nonlinear bounded function that takes into account the saturation phenomena for a large number of infectious. This approach is different than the classical mass action and standard incidence functions where the function is not bounded. Using an interaction term that tends to a saturation level is more realistic to model many diseases (Hethcote and van den Driessche 1991; Capasso and Serio 1978; Brauer 2017).

One way to see the interaction term is by means of the functional form $\operatorname{Sg}(I)$, where the function $g(I)$ is the force of infection. There are many functions that can be used for the function $g(I)$. For instance, $g(I)=\beta I$ would give a mass action transmission, which implies that the transmission rate increases with the size of the population, due to an increase of contacts with crowding. However, for some cases like diseases related to sexual contact, this assumption seems not realistic. Another situation that needs to be considered is that 
when there is a dangerous epidemic the people would react to reduce the number of contacts or even go to a self-quarantine to decrease the probability of infection (Capasso and Serio 1978; Hethcote and van den Driessche 1991). Thus, in some cases, it makes sense to assume a function $g(I)$ with a saturate level or even a function that decreases for large number of infectives due to an awareness in the population (Capasso and Serio 1978). Mathematical models have been published using different types of infection force (Hethcote and van den Driessche 1991)

In Guerrero-Flores et al. (2019), the authors present a family of epidemiological models of the SIRS form, which model the transmission of a fatal disease, which has seasonal variations and has a periodic contagion rate. In these models, the force of infection is given by a function $\beta(t) f(I)$ where $I$ represents the number of infectious people. Using LeraySchauder degree theory, they showed that the solutions are periodic depending on the value of the threshold parameter $R_{0}$. Other interesting works related to periodic solutions include seasonally forced $S I R$ and SEIRS models with impact of media coverage are presented in Zu and Wang (2015), Ávila-Vales et al. (2017).

When an epidemic model includes an incidence function that is nonlinear, usually, it presents complex structures which somehow reflect the effects of saturation. A typical functional form is the one based on the Holling type (Safi and Garba 2012), which is given by:

$$
f\left(I_{u}, I_{e}\right)=\beta \frac{I_{u}}{1+\alpha_{1} I_{u}}+\beta \frac{\eta I_{e}}{1+\alpha_{2} I_{e}},
$$

where $\beta$ includes the product of the contact rate and the probability of transmission given a contact. In addition, the parameter $\eta$ represents the effect of reducing the transmission of the disease by educated individuals compared to the class of the uneducated infectives. Thus, a minimum value of $\eta=0$ means that educated individuals would not be able to spread the disease, and a maximum value of $\eta=1$ means that both types of infectives transmit the disease with the same rate. Along these lines, a type of infection force has been defined as $g(S, I)=k S^{h} I /\left(S^{h}+\alpha I^{h}\right)$ (Ren and Zhang 2017; Ponciano and Capistran 2011).

For many diseases, there is an incubation period after the individual has an effective contact with an infected individual. That is, the action of the pathogen is not immediate, but after a period of time has elapsed. The symptoms of the disease appear after the incubation period, and then, latent individuals enter the infectious class.

One step further beside the introduction of the force of infection functions with saturation effect is the use of functions varying on time. For instance, in Mateus and Silva (2017), the authors presented a force of infection given by a function of the form $\beta(t) \phi(S, N, I)$. The authors presented the existence and stability analysis of endemic periodic solutions for a family of SEIRS models. Similar interesting models with some variants are also presented in Derrick and Van den Driessche (1993), Gao et al. (2018).

The force of infection is an inherent characteristic of each model, it has been generalized through different studies. In Luca et al. (2018), the authors proposed a force of infection for a susceptible individual with an age $i$, and given by the following expression:

$$
\lambda(i, p, t)=\beta \sum_{j} C_{i j}(t) \frac{I_{j}^{p}(t)}{N^{p}(t)},
$$

where $j$ runs on age classes, $I_{j}^{p}(t)$ and $N^{p}(t)$ count the total number of infectious individuals of age group $j$ and the total population size of patch $p$ at time $t$, respectively. Other interesting

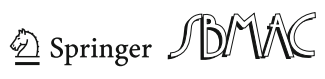


works along these lines can be seen in Korobeinikov (2009), Valle et al. (2013), Blackwood and Childs (2018) and Gölgeli and Atay (2020).

In this paper, we generalize different mathematical models presented previously and mentioned above. In particular, we study the existence and the analytical behavior of a $S_{i}, E_{i}, I_{i}, R_{i}$ type model. This model is based on a deterministic ordinary differential equation system, where the force of infection is given by a function $\left.\beta_{i}(t) f\left(I_{1}(t), I_{2}(t)\right)\right)$, which generalizes the models presented in Hogan et al. (2016b), White et al. (2005), White et al. (2007), Weber et al. (2001), Hogan et al. (2016a) and Hogan et al. (2017). We employ some interesting ideas used in related works in mathematical modeling in epidemiology (Luca et al. 2018; Capasso and Serio 1978; Guerrero-Flores et al. 2019; Mateus and Silva 2017; Valle et al. 2013; Blackwood and Childs 2018; Gölgeli and Atay 2020; Safi and Garba 2012; Jia and Zhang 2014). The generalized model that we propose is given by the following nonautonomous system:

$$
\begin{aligned}
\dot{S}_{1}(t) & =\mu-\beta_{1}(t) S_{1}(t) f\left(I_{1}(t), I_{2}(t)\right)+v R_{1}(t)-\eta S_{1}(t), \\
\dot{E}_{1}(t) & =\beta_{1}(t) S_{1}(t) f\left(I_{1}(t), I_{2}(t)\right)-\delta E_{1}(t)-\eta E_{1}(t), \\
\dot{I}_{1}(t) & =\delta E_{1}(t)-\gamma I_{1}(t)-\eta I_{1}(t), \\
\dot{R}_{1}(t) & =\gamma I_{1}(t)-v R_{1}(t)-\eta R_{1}(t), \\
\dot{S}_{2}(t) & =\eta S_{1}(t)-\beta_{2}(t) S_{2}(t) f\left(I_{1}(t), I_{2}(t)\right)+v R_{2}(t)-\eta S_{2}(t), \\
\dot{E}_{2}(t) & =\eta E_{1}(t)+\beta_{2}(t) S_{2}(t) f\left(I_{1}(t), I_{2}(t)\right)-\delta E_{2}(t)-\eta E_{2}(t), \\
\dot{I}_{2}(t) & =\eta I_{1}(t)+\delta E_{2}(t)-\gamma I_{2}(t)-\eta I_{2}(t), \\
\dot{R}_{2}(t) & =\eta R_{1}(t)+\gamma I_{2}(t)-v R_{2}(t)-\eta R_{2}(t),
\end{aligned}
$$

where $N_{i}(t)=S_{i}(t)+E_{i}(t)+I_{i}(t)+R_{i}(t)$ for $i=1,2$ and total population is given by $N(t)=N_{1}(t)+N_{2}(t)$. Moreover, the parameter $\mu$ is the birth rate, $\eta$ is the aging rate, $\beta_{1}(t), \beta_{2}(t)$ are T-periodic functions related to the transmission of the disease, $1 / \delta$ is the mean value of the latent period, $1 / \gamma$ is the mean value of the infectious period, and $1 / v$ is the mean value of the immunity period. The seasonal force of infection is given in terms of $\beta_{1}(t) f\left(I_{1}, I_{2}\right), \beta_{2}(t) f\left(I_{1}, I_{2}\right)$ where $\beta_{2}(t)=\alpha \beta_{1}(t)$ with $0<\alpha<1$, and $\alpha$ is a scale parameter for reduced susceptibility for the age class $i=2$ (older class). This type of infection force was presented in previous works (Blackwood and Childs 2018; Valle et al. 2013; Gölgeli and Atay 2020).

One important problem related to population dynamics is the study of epidemiological models in a seasonal environment. Thus, in this paper, we are interested in the analysis of the existence and global stability of positive periodic solutions. These last characteristics play a similar role as the globally stable equilibrium does in the autonomous models. Therefore, it is of paramount importance to find out the necessary conditions under which the nonautonomous system underlying the proposed mathematical model would present a positive periodic solution and to show that this particular solution is globally asymptotically stable.

We will start with some preliminary steps that will help to find the aforementioned conditions. First, since the model (3) is nonhomogeneous of degree one, and for the sake of clarity, we make the following change of variable:

$$
\begin{aligned}
& w_{1}(t)=S_{1}(t), \quad w_{2}(t)=E_{1}(t), \quad w_{3}(t)=I_{1}(t), \quad w_{4}(t)=R_{1}(t), \\
& w_{5}(t)=S_{2}(t), \quad w_{6}(t)=E_{2}(t), \quad w_{7}(t)=I_{2}(t), \quad w_{8}(t)=R_{2}(t),
\end{aligned}
$$


which that (3) can be written as:

$$
\begin{aligned}
& \dot{w}_{1}(t)=\mu-\beta_{1}(t) w_{1}(t) f\left(w_{3}(t), w_{7}(t)\right)+v w_{4}(t)-\eta w_{1}(t), \\
& \dot{w}_{2}(t)=\beta_{1}(t) w_{1}(t) f\left(w_{3}(t), w_{7}(t)\right)-\delta w_{2}(t)-\eta w_{2}(t), \\
& \dot{w}_{3}(t)=\delta w_{2}(t)-\gamma w_{3}(t)-\eta w_{3}(t), \\
& \dot{w}_{4}(t)=\gamma w_{3}(t)-v w_{4}(t)-\eta w_{4}(t), \\
& \dot{w}_{5}(t)=\eta w_{1}(t)-\beta_{2}(t) w_{5}(t) f\left(w_{3}(t), w_{7}(t)\right)+v w_{8}(t)-\eta w_{5}(t), \\
& \dot{w}_{6}(t)=\eta w_{2}(t)+\beta_{2}(t) w_{5}(t) f\left(w_{3}(t), w_{7}(t)\right)-\delta w_{6}(t)-\eta w_{6}(t), \\
& \dot{w}_{7}(t)=\eta w_{3}(t)+\delta w_{6}(t)-\gamma w_{7}(t)-\eta w_{7}(t), \\
& \dot{w}_{8}(t)=\eta w_{4}(t)+\gamma w_{7}(t)-v w_{8}(t)-\eta w_{8}(t) .
\end{aligned}
$$

This paper is organized in the following form. In Sect. 2, we present some notation and preliminary results for the sake of clarity. In the next Sect. 3, we prove the positivity and boundedness of the solutions. Our main results are presented in the next Sect. 4; we prove the existence of periodic solutions in terms of the related parameters of the model in a common interval period. In Sect. 5, we provide sufficient conditions that assure the global attractivity of the positive periodic solution. In Sect. 6, we conduct numerical simulations that support the theoretical results obtained. Finally, in Sect. 7, we draw the conclusions.

\section{Notation and preliminary results}

In this section, we will define some mathematical concepts. In addition, we will show some assumptions and establish some preliminary results. We define the following conventions:

$$
\bar{h}=\frac{1}{T} \int_{0}^{T} h(t) \mathrm{d} t, h^{u}=\max _{t \in[0, T]} h(t), h^{l}=\min _{t \in[0, T]} h(t),
$$

where $h(t)$ is a continuous $T$-periodic function. Next, the following hypotheses are assumed:

$\left[\mathrm{H}_{1}\right]$ The parameters: $\mu, \eta, \alpha, \delta, \gamma, v$ belong to $\left(0, M_{T}\right]$, where $M_{T}>0$.

$\left[\mathrm{H}_{2}\right]$ There is $T>0$, such that $\beta_{1}(t), \beta_{2}(t)$ are positive $T$-periodic continuous functions in $[0, T]$ and there are positive numbers $\beta_{1}^{u}, \beta_{1}^{l}$ with $\beta_{1}^{u} \geq \beta_{1}(t) \geq \beta_{1}^{l}$, and $\beta_{2}^{u} \geq$ $\beta_{2}(t) \geq \beta_{2}^{l}$.

$\left[\mathrm{H}_{3}\right] f:[0, \infty) \times[0, \infty) \rightarrow[0, \infty)$ is a differentiable function. $f(0,0)=0$, and $f(x, y)>0$, for all $x, y>0$. $\frac{\partial f(x, y)}{\partial x}, \frac{\partial f(x, y)}{\partial y}>0$, for all $x, y \geq 0$.

$\left[\mathrm{H}_{4}\right] x \frac{\partial f(x, y)}{\partial x}+y \frac{\partial f(x, y)}{\partial y}-f(x, y) \leq 0$, for all $x, y>0$.

$\left[\mathrm{H}_{5}\right]$ There exists a $\zeta_{2}>0$, such that for all $x, y>0$, it holds that: $0<f(x, y) \leq \zeta_{2}$.

From system (3), it can be seen that the hypothesis $\left[\mathrm{H}_{1}\right]$, is biologically correct, since these represent time lapses and contact rates. Moreover, the presumption for $\beta_{1}(t)$ and $\beta_{2}(t)$ in $\left[\mathrm{H}_{2}\right]$ is a natural way to introduce seasonality in these types of models (White et al. 2005; Hogan et al. 2016a; Arenas et al. 2008). Now, from condition $\left[\mathrm{H}_{3}\right]$, if there are no infectives, then there is no contact between susceptible and infectives, which is biologically natural for the this mathematical model (3).

After some calculations, it is verified that the class of functions $f\left(w_{3}, w_{7}\right)$ that represent the incidence rate, and that satisfy the above assumptions, are given by: $f\left(w_{3}, w_{7}\right)=a w_{3}+$ $b w_{7},(a, b>0)$, which is considered in Mitchell and Kribs (2019), Blackwood and Childs 
(2018), Arenas et al. (2008), Hogan et al. (2016a), Hogan et al. (2017), Gölgeli and Atay (2020), and in the same way those presented in Eq. (1) and in Eq. (2). Indeed, for the case equation (1), we have:

1. $f(0,0)=0, f\left(I_{u}, I_{e}\right)>0$, for all $I_{u}>0, I_{e}>0$,

2. $\frac{\partial f}{\partial I_{u}}\left(I_{u}, I_{e}\right)=\frac{\beta}{\left(1+\alpha_{1} I_{u}\right)^{2}}>0, \frac{\partial f}{\partial I_{e}}\left(I_{u}, I_{e}\right)=\frac{\beta \eta}{\left(1+\alpha_{2} I_{e}\right)^{2}}>0$, for all $I_{u}, I_{e} \geq 0$,

3. $\frac{I_{u} \beta}{\left(1+\alpha_{1} I_{u}\right)^{2}}+\frac{I_{e} \beta \eta}{\left(1+\alpha_{2} I_{e}\right)^{2}}-\frac{I_{u} \beta}{\left(1+\alpha_{1} I_{u}\right)}-\frac{I_{e} \beta \eta}{\left(1+\alpha_{2} I_{e}\right)} \leq 0$, for all $I_{u}, I_{e} \geq 0$,

4. $0<f\left(I_{u}, I_{e}\right)=\frac{\beta \alpha_{1} I_{u}}{\alpha_{1}\left(1+\alpha_{1} I_{u}\right)}+\frac{\beta \eta \alpha_{2} I_{e}}{\alpha_{2}\left(1+\alpha_{2} I_{e}\right)}<\frac{\beta}{\alpha_{1}}+\frac{\beta \eta}{\alpha_{2}}=\zeta_{2}$, for all $I_{u}, I_{e}>0$.

Another type of incidence functions less general than the bi-linear one that we use and present in Eq. (1) are functions of the form $f\left(w_{3}\right)=\beta w_{3} /\left(1+k w_{3}\right)$. These particular forms have been used to represent psychological or media effects for the infected population, and can be seen in Safi and Garba (2012), Guerrero-Flores et al. (2019).

\section{Positivity and boundedness of the solutions}

The model (4) must be consistent with the characteristics of a system of differential equations that represent a biologic population; in this case, the solution must be positive and bounded for all time. The following result shows these two characteristics.

Lemma 1 Assume $w(t)=\left(w_{1}(t), \ldots, w_{8}(t)\right)$ is a solution of system (4), such that $w_{i}(0)>0$ for all $i=1, \ldots, 8$, and $\left[\mathrm{H}_{1}\right]-\left[\mathrm{H}_{4}\right]$ hold. Then, $\left(w_{1}(t), \ldots, w_{8}(t)\right)$ is positive for all $t>0$ and ultimately bounded.

Proof Using the fact that the solution of system (4) is continuous, we can define:

$$
\mathfrak{M}(t)=\min _{t>0}\left\{w_{1}(t), \ldots, w_{8}(t)\right\}
$$

with $\mathfrak{M}(0)>0$. Now, if there exists a $t_{1}>0$, such that $\mathfrak{M}\left(t_{1}\right)=0, \mathfrak{M}(t)>0$ for $t \in\left[0, t_{1}\right)$, and we suppose that $\mathfrak{M}\left(t_{1}\right)=w_{1}\left(t_{1}\right)=0$ and $w_{4}(t)>0$ for $t \in\left[0, t_{1}\right)$. From the first equation of system (4), one gets:

$$
\dot{w}_{1}(t)>-\beta_{1}(t) w_{1}(t) f\left(w_{3}(t), w_{7}(t)\right)-\eta w_{1}(t),
$$

for $t \in\left[0, t_{1}\right]$. Therefore, applying the Comparison Principle in ordinary differential equation and using the continuity of $w_{1}(t)$ :

$$
0=w_{1}\left(t_{1}\right) \geq w_{1}(0) \exp \left(-\int_{0}^{t_{1}}\left(\beta_{1}(s) f\left(w_{3}(s), w_{7}(s)\right)-\eta\right) \mathrm{d} s\right)>0,
$$

which is a contradiction. Considering the cases for $\mathfrak{M}\left(t_{1}\right)=w_{2}\left(t_{1}\right)=\cdots=w_{8}\left(t_{1}\right)$, respectively, similar contradictions are deduced (Caraballo and Han 2017; Calatayud and Jornet 2020; Li et al. 2017). Thus, it follows that $\mathfrak{M}(t)>0$ for all $t>0$, i.e., $x_{i}(t)>0$ for all $t>0$ and $i=1, \ldots, 8$.

Next, to prove boundedness of system (4), first, we define:

$$
\mathscr{V}_{1}\left(w_{1}(t), \ldots, w_{8}(t)\right)=\sum_{i=1}^{4} w_{i}(t)+\frac{1}{2} \sum_{i=5}^{8} w_{i}(t)
$$


Then:

$$
\dot{\mathscr{V}}_{1}\left(w_{1}(t), \ldots, w_{8}(t)\right)=\sum_{i=1}^{4} \dot{w}_{i}(t)+\frac{1}{2} \sum_{i=5}^{8} \dot{w}_{i}(t)
$$

Thus:

$$
\dot{\mathscr{V}}_{1}<\mu-\frac{\eta}{2} \mathscr{V}_{1}
$$

Second, using the following function

$$
\mathscr{V}_{2}\left(w_{1}(t), \ldots, w_{8}(t)\right)=\sum_{i=1}^{4} w_{i}(t)+\frac{1}{4} \sum_{i=5}^{8} w_{i}(t)
$$

it follows that:

$$
\dot{\mathscr{V}}_{2}>\mu-\eta \mathscr{V} / 2
$$

By the theorem of differential inequality (Garrett Birkhoff 1989), one gets that:

$$
0<B^{l}:=\frac{\mu}{\eta} \leq \liminf _{t \longrightarrow \infty} \mathscr{V}_{2}(w(t))<\limsup _{t \rightarrow \infty} \mathscr{V}_{1}(w(t)) \leq \frac{2 \mu}{\eta}=: B^{u},
$$

as $t \longrightarrow \infty$. Thus, all the solutions of system (4) are ultimately bounded.

Remark 1 Moreover, from a biologically realistic point of view for sub-populations, for any $\epsilon>0$, there is $t_{\epsilon}>0$, such that for $t \geq t_{\epsilon}$, the set

$$
\mathscr{D}=\left\{\left(w_{1}(t), \ldots, w_{8}(t)\right) / 0<\frac{\mu}{\eta}-\epsilon \leq w_{i}(t) \leq \frac{2 \mu}{\eta}+\epsilon\right\} \subset \mathbb{R}_{+}^{8}
$$

is a positively invariant set for system (4).

\section{Existence of a positive periodic solutions}

The following results will be used to show the periodicity of the solutions of the mathematical model (4).

Proposition 1 If $\left[\mathrm{H}_{1}\right]-\left[\mathrm{H}_{5}\right]$ hold, then the system of algebraic equations:

$$
\begin{aligned}
& \mu e^{-\mathfrak{p}_{1}}-\overline{\beta_{1}} f\left(e^{\mathfrak{p}_{3}}, e^{\mathfrak{p}_{7}}\right)-\eta=0, \overline{\beta_{1}} e^{\mathfrak{p}_{1}-\mathfrak{p}_{2}} f\left(e^{\mathfrak{p}_{3}}, e^{\mathfrak{p}_{7}}\right)-\delta-\eta=0, \\
& \delta e^{\mathfrak{p}_{2}-\mathfrak{p}_{3}}-\gamma-\eta=0, \gamma e^{\mathfrak{p}_{3}-\mathfrak{p}_{4}}-v-\eta=0, \eta e^{\mathfrak{p}_{1}-\mathfrak{p}_{5}}-\overline{\beta_{2}} f\left(e^{\mathfrak{p}_{3}}, e^{\mathfrak{p}_{7}}\right)-\eta=0, \\
& \overline{\beta_{2}} e^{\mathfrak{p}_{5}-\mathfrak{p}_{6}} f\left(e^{\mathfrak{p}_{3}}, e^{\mathfrak{p}_{7}}\right)-\delta-\eta=0, \delta e^{\mathfrak{p}_{6}-\mathfrak{p}_{7}}-\gamma-\eta=0, \gamma e^{\mathfrak{p}_{7}-\mathfrak{p}_{8}}-v-\eta=0
\end{aligned}
$$

has a unique solution $e^{\mathfrak{p}_{1}}>0, e^{\mathfrak{p}_{2}}>0, \ldots, e^{\mathfrak{p}_{8}}>0$.

Proof It can be seen that the solutions are given by:

$$
\begin{aligned}
e^{\mathfrak{p}_{3}} & =\frac{\delta \mu}{(\delta+\mu)(\gamma+\eta)}>0, e^{\mathfrak{p}_{4}}=\frac{\gamma \delta \mu}{(\delta+\mu)(\gamma+\eta)}>0, \\
e^{\mathfrak{p}_{7}} & =\frac{\eta \delta e^{\mathfrak{p}_{1}}}{(\delta+\eta)(\gamma+\eta)}>0, e^{\mathfrak{p}_{8}}=\frac{\gamma \eta \delta e^{\mathfrak{p}_{1}}}{(\delta+\eta)(\gamma+\eta)(\nu+\eta)}>0 .
\end{aligned}
$$

For $e^{\mathfrak{p}_{1}}$, the function $g:\left[0, \frac{\mu}{\eta}\right] \longrightarrow R$, given by:

$$
g(t)=\mu-\overline{\beta_{1}} t f\left(\frac{\delta \mu}{(\delta+\mu)(\gamma+\eta)}, \frac{\eta \delta t}{(\delta+\eta)(\gamma+\eta)}\right)-\eta t
$$


is continuous and satisfies:

$$
g(0)=\mu>0,
$$

and

$$
g\left(\frac{\mu}{\eta}\right)=\mu-\overline{\beta_{1}} \frac{\mu}{\eta} f\left(\frac{\delta \mu}{(\delta+\mu)(\gamma+\eta)}, \frac{\delta \mu}{(\delta+\eta)(\gamma+\eta)}\right)-\mu<0 .
$$

By Bolzano's theorem, there is $\zeta \in] 0, \frac{\mu}{\eta}[$, such that $g(\zeta)=0$. Moreover:

$$
\begin{aligned}
g^{\prime}(t)= & -\overline{\beta_{1}} f\left(\frac{\delta \mu}{(\delta+\mu)(\gamma+\eta)}, \frac{\eta \delta t}{(\delta+\eta)(\gamma+\eta)}\right) \\
& -\overline{\beta_{1}} t \frac{\partial}{\partial w_{7}} f\left(\frac{\delta \mu}{(\delta+\mu)(\gamma+\eta)}, \frac{\eta \delta t}{(\delta+\eta)(\gamma+\eta)}\right) \frac{\eta \delta}{(\delta+\eta)(\gamma+\eta)}-\eta<0,
\end{aligned}
$$

for all $t \in] 0, \frac{\mu}{\eta}\left[\right.$. Thus, $\zeta$ is unique. Consequently, $e^{\mathfrak{p}_{1}}$ exists and, therefore, satisfies the second equation that combining with the first equation yields that $e^{\mathfrak{p}_{2}}=\frac{\mu-\eta e^{\mathfrak{p}_{1}}}{\delta+\eta}>0$. Thus:

$$
e^{\mathfrak{p}_{5}}=\frac{\eta e^{\mathfrak{p}_{1}}}{\overline{\beta_{2}} f\left(e^{\mathfrak{p}_{3}}, e^{\mathfrak{p}_{7}}\right)+\eta}>0, \quad e^{\mathfrak{p}_{6}}=\frac{\eta e^{\mathfrak{p}_{1}} \overline{\beta_{2}} f\left(e^{\mathfrak{p}_{3}}, e^{\mathfrak{p}_{7}}\right)}{(\delta+\eta)\left(\overline{\beta_{2}} f\left(e^{\mathfrak{p}_{3}}, e^{\mathfrak{p}_{7}}\right)+\eta\right)}>0,
$$

where $e^{\mathfrak{p}_{6}}$ results from combining equations five and six.

Lemma 2 Let $f$ be a function, such that $f(x) \geq 0$, integrable, and uniformly continuous on $[0,+\infty]$, and then, $\lim _{x \rightarrow \infty} f(x)=0$, see Samanta et al. (2015), Gopalsamy (2013).

One of the topological tools used as an alternative for the demonstration of the existence of periodic solutions is the well-known continuation theorem of Mawhin or degree of coincidence published by Jean Mawhin in the decade of the seventies.

In Mawhin (2005), it is shown how it was the origin of the degree of coincidence. The strength and usefulness of this theorem for seasonal epidemiological models is based on using the parameters of the model in a common periodical interval, which can be seen in the following references (Zhang et al. 2012; Gaines and Mawhin 2006; Jódar et al. 2008; Li et al. 2017; Wang 2015; Chen 2005; Li and Xiong 2010; Li and Qin 2018). On the other hand, using a novel similar continuation theorem of coincidence degree theory, some authors proved the existence of anti-periodic solutions ( $\mathrm{Li}$ et al. 2019).

Consider $\mathscr{X}$ and $\mathscr{Z}$ two normed and real spaces of infinite dimension. The linear operator $\mathscr{L}: \operatorname{Dom} \mathscr{L} \subset \mathscr{X} \longrightarrow \mathscr{Z}$; it is said of Fredholm of index zero if:

1. Dimension of $\operatorname{Ker} \mathscr{L}$ is finite, and

2. $\operatorname{Im} \mathscr{L}$ is closed in $\mathscr{Z}$ and has a finite co-dimension:

3. Index $\mathscr{L}=\operatorname{dim} \operatorname{Ker} \mathscr{L}-\operatorname{codim} \operatorname{Im} \mathscr{L}=0$.

This implies that there are continuous projectors $\mathscr{P}: \mathscr{X} \longrightarrow \mathscr{X}$ and $\mathscr{Q}: \mathscr{Z} \longrightarrow \mathscr{Z}$ with $\operatorname{Im} \mathscr{P}=\operatorname{Ker} \mathscr{L}, \operatorname{Im} \mathscr{L}=\operatorname{Ker} \mathscr{Q}=\operatorname{Im}(\mathscr{I}-\mathscr{Q})$. In addition, $\mathscr{X}=\operatorname{Ker} \mathscr{L} \oplus \operatorname{Ker} \mathscr{P}$ and $\mathscr{Z}=\operatorname{Im} \mathscr{L} \oplus \operatorname{Im} \mathscr{Q}$. Thus, the mapping $\mathscr{O}=\left.\mathscr{L}\right|_{\text {Dom } \mathscr{L} \cap \operatorname{Ker} \mathscr{P}}:(\mathscr{I}-\mathscr{P}) \mathscr{X} \longrightarrow \operatorname{Im} \mathscr{L}$ is invertible. Let $\mathscr{C}_{P}$ the inverse of $\mathscr{O}$. Let $\Omega_{0} \subset \mathscr{X}$ an open and bounded, the mapping $\mathscr{N}$ is called $\mathscr{L}$-compact on $\Omega_{0}$ if $\mathscr{Q} \mathscr{N}\left(\Omega_{0}\right)$ is bounded, and $\mathscr{C}_{P}(1-\mathscr{Q}) \mathscr{N}: \Omega_{0} \longrightarrow \mathscr{X}$ is compact.

Now, the following Mawhin's theorem will allow us to find sufficient conditions to prove the existence of at least one periodic positive solution of the model (4). 
Theorem 1 Let $\Omega_{0} \subset \mathscr{X}$ be an open-bounded set. Let $\mathscr{L}$ be a Fredholm mapping of index zero and $\mathscr{N}$ be $\mathscr{L}$-compact on $\bar{\Omega}_{0}$. Then, the equation $\mathscr{L} \mathrm{x}=\mathscr{N} \mathrm{x}$ has at least one solution in Dom $\mathscr{L} \cap \bar{\Omega}_{0}$ if it holds:

1. $\mathscr{L} \mathrm{x} \neq \lambda \mathscr{N} \mathrm{x}$, with $\lambda \in(0,1)$, and $\mathrm{x} \in \partial \Omega_{0} \cap \operatorname{Dom} \mathscr{L}$,

2. $\mathscr{Q} \mathscr{N} \mathrm{x} \neq 0, \mathrm{x} \in \partial \Omega_{0} \cap \operatorname{Ker} \mathscr{L}$,

3. $\operatorname{deg}\left\{\mathscr{J} \mathscr{Q} \mathscr{N}, \Omega_{0} \cap \operatorname{Ker} \mathscr{L}, 0\right\} \neq 0$.

One of the important results in this paper is the following:

Theorem 2 Suppose that $\left[\mathrm{H}_{1}\right]-\left[\mathrm{H}_{5}\right]$ hold, and then, there exists a positive T-periodic solution of model (4).

Proof Let us use the following change of variables:

$$
w_{i}(t)=e^{\mathfrak{u}_{\mathfrak{i}}(t)}, i=1, \ldots, 8 .
$$

Thus, (4) is equivalent to:

$$
\begin{aligned}
& \dot{\mathfrak{u}}_{1}(t)=\mu e^{-\mathfrak{u}_{1}(t)}-\beta_{1}(t) f\left(e^{\mathfrak{u}_{3}(t)}, e^{\mathfrak{u}_{7}(t)}\right)+v e^{\mathfrak{u}_{4}(t)-\mathfrak{u}_{1}(t)}-\eta, \\
& \dot{\mathfrak{u}}_{2}(t)=\beta_{1}(t) e^{\mathfrak{u}_{1}(t)-\mathfrak{u}_{2}(t)} f\left(e^{\mathfrak{u}_{3}(t)}, e^{\mathfrak{u}_{7}(t)}\right)-\delta-\eta, \\
& \dot{\mathfrak{u}}_{3}(t)=\delta e^{\mathfrak{u}_{2}(t)-\mathfrak{u}_{3}(t)}-\gamma-\eta, \\
& \dot{\mathfrak{u}}_{4}(t)=\gamma e^{\mathfrak{u}_{3}(t)-\mathfrak{u}_{4}(t)}-v-\eta, \\
& \dot{\mathfrak{u}}_{5}(t)=\eta e^{\mathfrak{u}_{1}(t)-\mathfrak{u}_{5}(t)}-\beta_{2}(t) f\left(e^{\mathfrak{u}_{3}(t)}, e^{\mathfrak{u}_{7}(t)}\right)+v e^{\mathfrak{u}_{8}(t)-\mathfrak{u}_{5}(t)}-\eta, \\
& \dot{\mathfrak{u}}_{6}(t)=\eta e^{\mathfrak{u}_{2}(t)-\mathfrak{u}_{6}(t)}+\beta_{2}(t) e^{\mathfrak{u}_{5}(t)-\mathfrak{u}_{6}(t)} f\left(e^{\mathfrak{u}_{3}(t)}, e^{\mathfrak{u}_{7}(t)}\right)-\delta-\eta, \\
& \dot{\mathfrak{u}}_{7}(t)=\eta e^{\mathfrak{u}_{3}(t)-\mathfrak{u}_{7}(t)}+\delta e^{\mathfrak{u}_{6}(t)-\mathfrak{u}_{7}(t)}-\gamma-\eta, \\
& \dot{\mathfrak{u}}_{8}(t)=\eta e^{\mathfrak{u}_{4}(t)-\mathfrak{u}_{8}(t)}+\gamma e^{\mathfrak{u}_{7}(t)-\mathfrak{u}_{8}(t)}-v-\eta .
\end{aligned}
$$

Now, suppose that one solution $\left(\mathfrak{u}_{1}^{*}(t), \ldots, \mathfrak{u}_{8} *(t)\right)^{T}$, of $(8)$ is positive $T$-periodic, and then, $\left(w_{1}^{*}(t), \ldots, w_{8}^{*}(t)\right)^{T}$, is a positive $T$-periodic solution of the system (4). This implies that there exists one solution of system (3). Therefore, it is sufficient to show that the system (8) has at least one positive T-periodic solution. To verify the hypotheses of Theorem 1, we define the following spaces:

$$
\mathscr{X}=\mathscr{Y}=\left\{\mathfrak{u}(t)=\left(\mathfrak{u}_{1}(t), \ldots, \mathfrak{u}_{8}(t)\right)^{T} \in C\left(\mathbb{R}, \mathbb{R}^{8}\right) / \mathfrak{u}(T+t)=\mathfrak{u}(t)\right\}
$$

with norm

$$
\|\mathfrak{u}\|=\sum_{i=1}^{8} \max _{t \in[0, T]}\left|\mathfrak{u}_{\mathfrak{i}}(t)\right| .
$$

It is easy to verify that $(\mathscr{X},\|\cdot\|)$ is a Banach space, Ding and Zhao (2012), Rui et al. (2004).

We define $\mathscr{L}: \operatorname{Dom} \mathscr{L} \cap \mathscr{X} \longrightarrow \mathscr{X}$, by $\mathscr{L}(\mathfrak{u}(t))=\dot{\mathfrak{u}}(t)=\frac{\mathrm{d} \mathfrak{u}(t)}{\mathrm{d} t}$, with:

$$
\operatorname{Dom} \mathscr{L}=\left\{\mathfrak{u}(t) \in C^{1}\left(\mathbb{R}, \mathbb{R}^{8}\right) / \mathfrak{u}(T+t)=\mathfrak{u}(t)\right\} \subseteq \mathscr{X},
$$


and $\mathscr{N}: \mathscr{X} \longrightarrow \mathscr{X}$ is:

$$
\mathscr{N} \mathfrak{u}(t)=\left[\begin{array}{l}
\mu e^{-\mathfrak{u}_{1}(t)}-\beta_{1}(t) f\left(e^{\mathfrak{u}_{3}(t)}, e^{\mathfrak{u}_{7}(t)}\right)+v e^{\mathfrak{u}_{4}(t)-\mathfrak{u}_{1}(t)}-\eta \\
\beta_{1}(t) e^{\mathfrak{u}_{1}(t)-\mathfrak{u}_{2}(t)} f\left(e^{\mathfrak{u}_{3}(t)}, e^{\mathfrak{u}_{7}(t)}\right)-\delta-\eta \\
\delta e^{\mathfrak{u}_{2}(t)-\mathfrak{u}_{3}(t)}-\gamma-\eta \\
\gamma e^{\mathfrak{u}_{3}(t)-\mathfrak{u}_{4}(t)}-v-\eta \\
\eta e^{\mathfrak{u}_{1}(t)-\mathfrak{u}_{5}(t)}-\beta_{2}(t) f\left(e^{\mathfrak{u}_{3}(t)}, e^{\mathfrak{u}_{7}(t)}\right)+v e^{\mathfrak{u}_{8}(t)-\mathfrak{u}_{5}(t)}-\eta \\
\eta e^{\mathfrak{u}_{2}(t)-\mathfrak{u}_{6}(t)}+\beta_{2}(t) e^{\mathfrak{u}_{5}(t)-\mathfrak{u}_{6}(t)} f\left(e^{\mathfrak{u}_{3}(t)}, e^{\mathfrak{u}_{7}(t)}\right)-\delta-\eta \\
\eta e^{\mathfrak{u}_{3}(t)-\mathfrak{u}_{7}(t)}+\delta e^{\mathfrak{u}_{6}(t)-\mathfrak{u}_{7}(t)}-\gamma-\eta \\
\eta e^{\mathfrak{u}_{4}(t)-\mathfrak{u}_{8}(t)}+\gamma e^{\mathfrak{u}_{7}(t)-\mathfrak{u}_{8}(t)}-v-\eta
\end{array}\right] .
$$

Put the projectors $\mathscr{P}: \mathscr{X} \longrightarrow \mathscr{X}$ and $\mathscr{Q}: \mathscr{Y} \longrightarrow \mathscr{Y}$ defined by:

$$
\mathscr{P} \mathfrak{u}(t)=\mathscr{Q} \mathfrak{u}(t)=\frac{1}{T} \int_{0}^{T} \mathfrak{u}(t) \mathrm{d} t .
$$

It follows that $\operatorname{Ker} \mathscr{L}=\mathbb{R}^{8}$, and:

$$
\operatorname{Im} \mathscr{L}=\operatorname{Ker} \mathscr{Q}=\operatorname{Im}(\mathscr{I}-\mathscr{Q})=\left\{\mathfrak{u} \in \mathscr{X} / \frac{1}{T} \int_{0}^{T} \mathfrak{u}(t) \mathrm{d} t=0\right\}
$$

is clearly closed in $\mathscr{X}$. In addition, Index $\mathscr{L}=0$, since $\operatorname{dim} \operatorname{Ker} \mathscr{L}=\operatorname{codimIm} \mathscr{L}=8$. Accordingly, $\mathscr{L}$ is a Fredholm mapping of index zero.

Now, after some calculations, we can see that:

$$
\mathscr{O}_{P}=\left.\mathscr{L}\right|_{D o m} \mathscr{L} \cap \operatorname{Ker} \mathscr{P}:(\mathscr{I}-\mathscr{P}) \mathscr{X} \longrightarrow \operatorname{Im} \mathscr{L}
$$

is invertible, and its inverse (to $\mathscr{O}_{P}$ ), $\mathscr{C}_{P}: \operatorname{Im} \mathscr{L} \longrightarrow D o m \mathscr{L} \cap \operatorname{Ker} \mathscr{P}$, is given by:

$$
\mathscr{C}_{P}(\mathfrak{u})=\int_{0}^{t} \mathfrak{u}(s) \mathrm{d} s-\frac{1}{T} \int_{0}^{T} \int_{0}^{t} \mathfrak{u}(s) \mathrm{d} s \mathrm{~d} t, \quad t \in[0, T] .
$$

Thus, $\mathscr{Q} \mathscr{N}: \mathscr{X} \longrightarrow \mathscr{X}$ is:

$$
\begin{aligned}
& \mathscr{Q} \mathscr{N} \mathfrak{u}(t)=\mathscr{Q}\left[\begin{array}{c}
\mu e^{-\mathfrak{u}_{1}(t)}-\beta_{1}(t) f\left(e^{\mathfrak{u}_{3}(t)}, e^{\mathfrak{u}_{7}(t)}\right)+v e^{\mathfrak{u}_{4}(t)-\mathfrak{u}_{1}(t)}-\eta \\
\beta_{2}(t) e^{\mathfrak{u}_{1}(t)-\mathfrak{u}_{2}(t)} f\left(e^{\mathfrak{u}_{3}(t)}, e^{\mathfrak{u}_{7}(t)}\right)-\delta-\eta \\
\delta e^{\mathfrak{u}_{2}(t)-\mathfrak{u}_{3}(t)}-\gamma-\eta \\
\gamma e^{\mathfrak{u}_{3}(t)-\mathfrak{u}_{4}(t)}-v-\eta \\
\eta e^{\mathfrak{u}_{1}(t)-\mathfrak{u}_{5}(t)}-\beta_{2}(t) f\left(e^{\mathfrak{u}_{3}(t)}, e^{\mathfrak{u}_{7}(t)}\right)+v e^{\mathfrak{u}_{8}(t)-\mathfrak{u}_{5}(t)}-\eta \\
\eta e^{\mathfrak{u}_{2}(t)-\mathfrak{u}_{6}(t)}+\beta_{2}(t) e^{\mathfrak{u}_{5}(t)-\mathfrak{u}_{6}(t)} f\left(e^{\mathfrak{u}_{3}(t)}, e^{\mathfrak{u}_{7}(t)}\right)-\delta-\eta \\
\eta e^{\mathfrak{u}_{3}(t)-\mathfrak{u}_{7}(t)}+\delta e^{\mathfrak{u}_{6}(t)-\mathfrak{u}_{7}(t)}-\gamma-\eta \\
\eta e^{\mathfrak{u}_{4}(t)-\mathfrak{u}_{8}(t)}+\gamma e^{\mathfrak{u}_{7}(t)-\mathfrak{u}_{8}(t)}-v-\eta
\end{array}\right] \\
& =\left[\begin{array}{l}
\frac{\mu}{T} \int_{0}^{T} e^{-\mathfrak{u}_{1}(t)} \mathrm{d} t-\frac{1}{T} \int_{0}^{T} \beta_{1}(t) f\left(e^{\mathfrak{u}_{3}(t)}, e^{\mathfrak{u}_{7}(t)}\right) \mathrm{d} t+\frac{\nu}{T} \int_{0}^{T} e^{\mathfrak{u}_{4}(t)-\mathfrak{u}_{1}(t)} \mathrm{d} t-\eta \\
\frac{1}{T} \int_{0}^{T} \beta_{1}(t) e^{\mathfrak{u}_{1}(t)-\mathfrak{u}_{2}(t)} f\left(e^{\mathfrak{u}_{3}(t)}, e^{\mathfrak{u}_{7}(t)}\right) \mathrm{d} t-\delta-\eta \\
\frac{\delta}{T} \int_{0}^{T} e^{\mathfrak{u}_{2}(t)-\mathfrak{u}_{3}(t)} \mathrm{d} t-\gamma-\eta \\
\frac{\gamma}{T} \int_{0}^{T} e^{\mathfrak{u}_{3}(t)-\mathfrak{u}_{4}(t)} \mathrm{d} t-v-\eta \\
\frac{\eta}{T} \int_{0}^{T} e^{\mathfrak{u}_{1}(t)-\mathfrak{u}_{5}(t)} \mathrm{d} t-\frac{1}{T} \int_{0}^{T} \beta_{1}(t) f\left(e^{\mathfrak{u}_{3}(t)}, e^{\mathfrak{u}_{7}(t)}\right) \mathrm{d} t+\frac{\nu}{T} \int_{0}^{T} e^{\mathfrak{u}_{8}(t)-\mathfrak{u}_{5}(t)} \mathrm{d} t-\eta \\
\frac{\eta}{T} \int_{0}^{T} e^{\mathfrak{u}_{2}(t)-\mathfrak{u}_{6}(t)} \mathrm{d} t+\frac{1}{T} \int_{0}^{T} \beta_{2}(t) e^{\mathfrak{u}_{5}(t)-\mathfrak{u}_{6}(t)} f\left(e^{\mathfrak{u}_{3}(t)}, e^{\mathfrak{u}_{7}(t)}\right) \mathrm{d} t-\delta-\eta \\
\frac{\eta}{T} \int_{0}^{T} e^{\mathfrak{u}_{3}(t)-\mathfrak{u}_{7}(t)} \mathrm{d} t+\frac{\delta}{T} \int_{0}^{T} e^{\mathfrak{u}_{6}(t)-\mathfrak{u}_{7}(t)} \mathrm{d} t-\gamma-\eta \\
\frac{\eta}{T} \int_{0}^{T} e^{\mathfrak{u}_{4}(t)-\mathfrak{u}_{8}(t)} \mathrm{d} t+\frac{\gamma}{T} \int_{0}^{T} e^{\mathfrak{u}_{7}(t)-\mathfrak{u}_{8}(t)} \mathrm{d} t-v-\eta
\end{array}\right] .
\end{aligned}
$$


The mapping $\mathscr{C}_{P}(\mathscr{I}-\mathscr{Q}) \mathscr{N}: \mathscr{X} \longrightarrow \mathscr{X}$ has the form:

$$
\mathscr{C}_{P}(\mathscr{I}-\mathscr{Q}) \mathscr{N} \mathfrak{u}(t)=\int_{0}^{t} \mathscr{N} \mathfrak{u}(s) \mathrm{d} s-\frac{1}{T} \int_{0}^{T} \int_{0}^{t} \mathscr{N} \mathfrak{u}(s) \mathrm{d} s \mathrm{~d} t-\left(\frac{t}{T}-\frac{1}{2}\right) \int_{0}^{T} \mathscr{N} \mathfrak{u}(s) \mathrm{d} s .
$$

Moreover, this mapping $\mathscr{Q} \mathscr{N}$ and $\mathscr{C}_{P}(\mathscr{I}-\mathscr{Q}) \mathscr{N}$ are continuous by the Lebesgue's convergence theorem, Chen (2005). If $\Omega_{0} \subset \mathscr{X}$ is a open-bounded set by Arzela-Ascoli's theorem, $\overline{\mathscr{C}_{P}(\mathscr{I}-\mathscr{Q}) \mathscr{N}\left(\overline{\Omega_{0}}\right)}$ are relatively compact. Moreover, $\mathscr{Q} \mathscr{N}\left(\overline{\Omega_{0}}\right)$ is bounded. Thus, $\mathscr{N}$ is $\mathscr{L}$-compact under $\overline{\Omega_{0}}$ with any open-bounded set $\Omega_{0} \subset \mathscr{X}$. The isomorphism $\mathscr{J}$ from $\operatorname{Im} \mathscr{Q}$ under $\operatorname{Ker} \mathscr{L}$ can be the identity mapping, since that $\operatorname{Im} \mathscr{Q}=\operatorname{Ker} \mathscr{L}$.

To verify the hypothesis of Theorem 1 , an open-bounded subset $\Omega_{0}$ must be constructed in the Banach $\mathscr{X}$ space. Thus, for the operator equation, $\mathscr{L} \mathfrak{u}=\lambda \mathscr{N} \mathfrak{u}$ with $\lambda \in(0,1)$, we have:

$$
\begin{aligned}
& \dot{\mathfrak{u}}_{1}(t)=\lambda\left(\mu e^{-\mathfrak{u}_{1}(t)}-\beta_{1}(t) f\left(e^{\mathfrak{u}_{3}(t)}, e^{\mathfrak{u}_{7}(t)}\right)+v e^{\mathfrak{u}_{4}(t)-\mathfrak{u}_{1}(t)}-\eta\right), \\
& \dot{\mathfrak{u}}_{2}(t)=\lambda\left(\beta_{1}(t) e^{\mathfrak{u}_{1}(t)-\mathfrak{u}_{2}(t)} f\left(e^{\mathfrak{u}_{3}(t)}, e^{\mathfrak{u}_{7}(t)}\right)-\delta-\eta\right), \\
& \dot{\mathfrak{u}}_{3}(t)=\lambda\left(\delta e^{\mathfrak{u}_{2}(t)-\mathfrak{u}_{3}(t)}-\gamma-\eta\right), \\
& \dot{\mathfrak{u}}_{4}(t)=\lambda\left(\gamma e^{\mathfrak{u}_{3}(t)-\mathfrak{u}_{4}(t)}-v-\eta\right), \\
& \dot{\mathfrak{u}}_{5}(t)=\lambda\left(\eta e^{\mathfrak{u}_{1}(t)-\mathfrak{u}_{5}(t)}-\beta_{2}(t) f\left(e^{\mathfrak{u}_{3}(t)}, e^{\mathfrak{u}_{7}(t)}\right)+v e^{\mathfrak{u}_{8}(t)-\mathfrak{u}_{5}(t)}-\eta\right), \\
& \dot{\mathfrak{u}}_{6}(t)=\lambda\left(\eta e^{\mathfrak{u}_{2}(t)-\mathfrak{u}_{6}(t)}+\beta_{2}(t) e^{\mathfrak{u}_{5}(t)-\mathfrak{u}_{6}(t)} f\left(e^{\mathfrak{u}_{3}(t)}, e^{\mathfrak{u}_{7}(t)}\right)-\delta-\eta\right), \\
& \dot{\mathfrak{u}}_{7}(t)=\lambda\left(\eta e^{\mathfrak{u}_{3}(t)-\mathfrak{u}_{7}(t)}+\delta e^{\mathfrak{u}_{6}(t)-\mathfrak{u}_{7}(t)}-\gamma-\eta\right), \\
& \dot{\mathfrak{u}}_{8}(t)=\lambda\left(\eta e^{\mathfrak{u}_{4}(t)-\mathfrak{u}_{8}(t)}+\gamma e^{\mathfrak{u}_{7}(t)-\mathfrak{u}_{8}(t)}-v-\eta\right) .
\end{aligned}
$$

Suppose that $\mathfrak{u}(t)=\left(\mathfrak{u}_{\mathfrak{1}}(t), \ldots, \mathfrak{u}_{8}(t)\right)^{T} \in \mathscr{X}$ is any solution of system (9) for a certain $\lambda \in(0,1)$. Integrating (9) over the interval $[0, T]$ leads to:

$$
\begin{aligned}
& \mu \int_{0}^{T} e^{-\mathfrak{u}_{1}(t)} \mathrm{d} t+v \int_{0}^{T} e^{\mathfrak{u}_{4}(t)-\mathfrak{u}_{1}(t)} \mathrm{d} t=\int_{0}^{T} \beta_{1}(t) f\left(e^{\mathfrak{u}_{3}(t)}, e^{\mathfrak{u}_{7}(t)}\right) \mathrm{d} t+\eta T . \\
& \int_{0}^{T} \beta_{1}(t) e^{\mathfrak{u}_{1}(t)-\mathfrak{u}_{2}(t)} f\left(e^{\mathfrak{u}_{3}(t)}, e^{\mathfrak{u}_{7}(t)}\right) \mathrm{d} t=(\delta+\eta) T \\
& \delta \int_{0}^{T} e^{\mathfrak{u}_{2}(t)-\mathfrak{u}_{3}(t)} \mathrm{d} t=(\gamma+\eta) T . \\
& \gamma \int_{0}^{T} e^{\mathfrak{u}_{3}(t)-\mathfrak{u}_{4}(t)} \mathrm{d} t=(v+\eta) T . \\
& \eta \int_{0}^{T} e^{\mathfrak{u}_{1}(t)-\mathfrak{u}_{5}(t)}+v \int_{0}^{T} e^{\mathfrak{u}_{8}(t)-\mathfrak{u}_{5}(t)} \mathrm{d} t=\int_{0}^{T} \beta_{2}(t) f\left(e^{\mathfrak{u}_{3}(t)}, e^{\mathfrak{u}_{7}(t)}\right) \mathrm{d} t+\eta T . \\
& \eta \int_{0}^{T} e^{\mathfrak{u}_{2}(t)-\mathfrak{u}_{6}(t)} \mathrm{d} t+\int_{0}^{T} \beta_{2}(t) e^{\mathfrak{u}_{5}(t)-\mathfrak{u}_{6}(t)} f\left(e^{\mathfrak{u}_{3}(t)}, e^{\mathfrak{u}_{7}(t)}\right) \mathrm{d} t=(\delta+\eta) T . \\
& \eta \int_{0}^{T} e^{\mathfrak{u}_{3}(t)-\mathfrak{u}_{7}(t)} \mathrm{d} t+\delta \int_{0}^{T} e^{\mathfrak{u}_{6}(t)-\mathfrak{u}_{7}(t)} \mathrm{d} t=(\gamma+\eta) T \\
& \eta \int_{0}^{T} e^{\mathfrak{u}_{4}(t)-\mathfrak{u}_{8}(t)} \mathrm{d} t+\gamma \int_{0}^{T} e^{\mathfrak{u}_{7}(t)-\mathfrak{u}_{8}(t)} \mathrm{d} t=(v+\eta) .
\end{aligned}
$$


It follows from (10)-(17) that:

$$
\begin{aligned}
& \int_{0}^{T}\left|\dot{\mathfrak{u}}_{1}(t)\right| \mathrm{d} t \leq \lambda\left(\mu \int_{0}^{T} e^{-\mathfrak{u}_{1}(t)} \mathrm{d} t+\int_{0}^{T} \beta_{1}(t) f\left(e^{\mathfrak{u}_{3}(t)}, e^{\mathfrak{u}_{7}(t)}\right) \mathrm{d} t\right. \\
& \left.+v \int_{0}^{T} e^{\mathfrak{u}_{4}(t)-\mathfrak{u}_{1}(t)} \mathrm{d} t+\eta T\right) \\
& <2\left(\int_{0}^{T} \beta_{1}(t) f\left(e^{\mathfrak{u}_{3}(t)}, e^{\mathfrak{u}_{7}(t)}\right) \mathrm{d} t+\eta T\right) \\
& <2\left(\zeta_{2} T \overline{\beta_{1}}+\eta T\right):=k_{1} \text {. } \\
& \int_{0}^{T}\left|\dot{\mathfrak{u}}_{2}(t)\right| \mathrm{d} t \leq \lambda\left(\int_{0}^{T} \beta_{1}(t) e^{\mathfrak{u}_{1}(t)-\mathfrak{u}_{2}(t)} f\left(e^{\mathfrak{u}_{3}(t)}, e^{\mathfrak{u}_{7}(t)}\right) \mathrm{d} t+(\delta+\eta) T\right) \\
& <2(\delta+\eta) T:=k_{2} \text {. } \\
& \int_{0}^{T}\left|\dot{\mathfrak{u}}_{3}(t)\right| \mathrm{d} t \leq \lambda\left(\delta \int_{0}^{T} e^{\mathfrak{u}_{2}(t)-\mathfrak{u}_{3}(t)} \mathrm{d} t+(\gamma+\eta) T\right) \\
& <2(\gamma+\eta) T:=k_{3} \text {. } \\
& \int_{0}^{T}\left|\dot{\mathfrak{u}}_{4}(t)\right| \mathrm{d} t \leq \lambda\left(\gamma \int_{0}^{T} e^{\mathfrak{u}_{3}(t)-\mathfrak{u}_{4}(t)} \mathrm{d} t+(\nu+\eta) T\right) \\
& <2(v+\eta) T:=k_{4} \text {. } \\
& \int_{0}^{T}\left|\dot{\mathfrak{u}}_{5}(t)\right| \mathrm{d} t \leq \lambda\left(\eta \int_{0}^{T} e^{\mathfrak{u}_{1}(t)-\mathfrak{u}_{5}(t)} \mathrm{d} t+\int_{0}^{T} \beta_{2}(t) f\left(e^{\mathfrak{u}_{3}(t)}, e^{\mathfrak{u}_{7}(t)}\right) \mathrm{d} t\right. \\
& \left.+v \int_{0}^{T} e^{\mathfrak{u}_{8}(t)-\mathfrak{u}_{5}(t)} \mathrm{d} t+\eta T\right) \\
& <2\left(\int_{0}^{T} \beta_{2}(t) f\left(e^{\mathfrak{u}_{3}(t)}, e^{\mathfrak{u}_{7}(t)}\right) \mathrm{d} t+\eta T\right) \\
& <2\left(\zeta_{2} T \overline{\beta_{2}}+\eta T\right):=k_{5} \text {. } \\
& \int_{0}^{T}\left|\dot{\mathfrak{u}}_{6}(t)\right| \mathrm{d} t \leq \lambda\left(\int_{0}^{T} \beta_{2}(t) e^{\mathfrak{u}_{5}(t)-\mathfrak{u}_{6}(t)} f\left(e^{\mathfrak{u}_{3}(t)}, e^{\mathfrak{u}_{7}(t)}\right) \mathrm{d} t+(\delta+\eta) T\right. \\
& \left.+\eta \int_{0}^{T} e^{\mathfrak{u}_{2}(t)-\mathfrak{u}_{6}(t)} \mathrm{d} t\right)<2(\delta+\eta) T:=k_{6} . \\
& \int_{0}^{T}\left|\dot{\mathfrak{u}}_{7}(t)\right| \mathrm{d} t \leq \lambda\left(\eta \int_{0}^{T} e^{\mathfrak{u}_{3}(t)-\mathfrak{u}_{7}(t)} \mathrm{d} t+\delta \int_{0}^{T} e^{\mathfrak{u}_{6}(t)-\mathfrak{u}_{7}(t)} \mathrm{d} t+(\gamma+\eta) T\right) \\
& <2(\gamma+\eta) T:=k_{7} \text {. } \\
& \int_{0}^{T}\left|\dot{\mathfrak{u}}_{8}(t)\right| \mathrm{d} t \leq \lambda\left(\eta \int_{0}^{T} e^{\mathfrak{u}_{4}(t)-\mathfrak{u}_{8}(t)} \mathrm{d} t+\gamma \int_{0}^{T} e^{\mathfrak{u}_{7}(t)-\mathfrak{u}_{8}(t)} \mathrm{d} t+(v+\eta) T\right) \\
& <2(v+\eta) T:=k_{8} \text {. }
\end{aligned}
$$

We are considering the solution $\mathfrak{u}(t) \in \mathscr{X}$ in the interval $[0, T]$. Then, there exist $\xi_{i}, \eta_{i} \in$ $[0, T]$ for $i=1, \ldots, 8$, such that:

$$
\mathfrak{u}_{\mathfrak{i}}\left(\xi_{i}\right)=\min _{t \in[0, T]} \mathfrak{u}_{\mathfrak{i}}(t), \quad \mathfrak{u}_{\mathfrak{i}}\left(\eta_{i}\right)=\max _{t \in[0, T]} \mathfrak{u}_{\mathfrak{i}}(t)
$$


To obtain upper and lower bounds of $\mathfrak{u}_{1}(t), \ldots, \mathfrak{u}_{8}(t)$, we multiply by $e^{\mathfrak{u}_{\mathfrak{i}}(t)}$ the $i$ th equation of system (9) (for $i=1, \ldots, 8$ ) and integrate over $[0, T]$. Thus:

$$
\begin{aligned}
& \mu T+v \int_{0}^{T} e^{\mathfrak{u}_{4}(t)} \mathrm{d} t+\int_{0}^{T} \beta_{1}(t) e^{\mathfrak{u}_{1}(t)} f\left(e^{\mathfrak{u}_{3}(t)}, e^{\mathfrak{u}_{7}(t)}\right) \mathrm{d} t=\eta \int_{0}^{T} e^{\mathfrak{u}_{1}(t)} \mathrm{d} t, \\
& \int_{0}^{T} \beta_{1}(t) e^{\mathfrak{u}_{1}(t)} f\left(e^{\mathfrak{u}_{3}(t)}, e^{\mathfrak{u}_{7}(t)}\right) \mathrm{d} t=(\delta+\eta) \int_{0}^{T} e^{\mathfrak{u}_{2}(t)} \mathrm{d} t, \\
& \delta \int_{0}^{T} e^{\mathfrak{u}_{2}(t)} \mathrm{d} t=(\gamma+\eta) \int_{0}^{T} e^{\mathfrak{u}_{3}(t)} \mathrm{d} t, \\
& \gamma \int_{0}^{T} e^{\mathfrak{u}_{3}(t)} \mathrm{d} t=(v+\eta) \int_{0}^{T} e^{\mathfrak{u}_{4}(t)} \mathrm{d} t, \\
& \eta \int_{0}^{T} e^{\mathfrak{u}_{1}(t)} \mathrm{d} t+v \int_{0}^{T} e^{\mathfrak{u}_{8}(t)} \mathrm{d} t=\int_{0}^{T} \beta_{2}(t) e^{\mathfrak{u}_{5}(t)} f\left(e^{\mathfrak{u}_{3}(t)}, e^{\mathfrak{u}_{7}(t)}\right) \mathrm{d} t \\
& \quad+\eta \int_{0}^{T} e^{\mathfrak{u}_{5}(t)} \mathrm{d} t, \\
& \eta \int_{0}^{T} e^{\mathfrak{u}_{2}(t)} \mathrm{d} t+\int_{0}^{T} \beta_{2}(t) e^{\mathfrak{u}_{5}(t)} f\left(e^{\mathfrak{u}_{3}(t)}, e^{\mathfrak{u}_{7}(t)}\right) \mathrm{d} t=(\delta+\eta) \int_{0}^{T} e^{\mathfrak{u}_{6}(t)} \mathrm{d} t, \\
& \eta \int_{0}^{T} e^{\mathfrak{u}_{3}(t)} \mathrm{d} t+\delta \int_{0}^{T} e^{\mathfrak{u}_{6}(t)} \mathrm{d} t=(\gamma+\eta) \int_{0}^{T} e^{\mathfrak{u}_{7}(t)} \mathrm{d} t, \\
& \eta \int_{0}^{T} e^{\mathfrak{u}_{4}(t)} \mathrm{d} t+\gamma \int_{0}^{T} e^{\mathfrak{u}_{7}(t)} \mathrm{d} t=(v+\eta) \int_{0}^{T} e^{\mathfrak{u}_{8}(t)} \mathrm{d} t .
\end{aligned}
$$

Then, from Eq. (26a), we have that:

$$
\mu T<(\eta+\mu) \int_{0}^{T} e^{\mathfrak{u}_{1}(t)} \mathrm{d} t \leq e^{\mathfrak{u}_{1}\left(\eta_{1}\right)}(\eta+\mu) T .
$$

Therefore:

$$
e^{\mathfrak{u}_{1}\left(\eta_{1}\right)}>\frac{\mu}{\mu+\eta}:=B_{1} .
$$

Now, from Eq. (26b), there is $\theta_{1}^{*} \in[0, T]$, such that:

$$
\begin{aligned}
T M & :=T \overline{\beta_{1}} e^{\mathfrak{u}_{1}\left(\theta_{1}^{*}\right)} f\left(e^{\mathfrak{u}_{3}\left(\theta_{1}^{*}\right)}, e^{\mathfrak{u}_{7}\left(\theta_{1}^{*}\right)}\right)=\frac{1}{T} \int_{0}^{T} \beta_{1}(t) e^{\mathfrak{u}_{1}(t)} f\left(e^{\mathfrak{u}_{3}(t)}, e^{\mathfrak{u}_{7}(t)}\right) \mathrm{d} t \\
& =(\delta+\eta) \int_{0}^{T} e^{\mathfrak{u}_{2}(t)} \mathrm{d} t<(\delta+\eta+M) e^{\mathfrak{u}_{2}\left(\eta_{2}\right)} T .
\end{aligned}
$$

Thus:

$$
e^{\mathfrak{u}_{2}\left(\eta_{2}\right)}>\frac{M}{\delta+\eta+M}:=B_{2} .
$$

From (26c), there exists $\theta_{2}^{*} \in[0, T]$, such that:

$$
\begin{aligned}
\delta T e^{\mathfrak{u}_{2}\left(\theta_{2}^{*}\right)} & =\delta \int_{0}^{T} e^{\mathfrak{u}_{2}(t)} \mathrm{d} t \\
& =(\gamma+\eta) \int_{0}^{T} e^{\mathfrak{u}_{3}(t)} \mathrm{d} t<\left(e^{\mathfrak{u}_{2}\left(\theta_{2}^{*}\right)} \delta+\gamma+\eta\right) e^{\mathfrak{u}_{3}\left(\eta_{3}\right)} T .
\end{aligned}
$$


In this way:

$$
e^{\mathfrak{u}_{3}\left(\eta_{3}\right)}>\frac{\delta e^{\mathfrak{u}_{2}\left(\theta_{2}^{*}\right)}}{\delta e^{\mathfrak{u}_{2}\left(\theta_{2}^{*}\right)}+\gamma+\eta}:=B_{3} .
$$

Next, there exists a $\theta_{3}^{*} \in[0, T]$ in the Eq. (26d), such that:

$$
T \gamma e^{\mathfrak{u}_{3}\left(\theta_{3}^{*}\right)}=\gamma \int_{0}^{T} e^{\mathfrak{u}_{3}(t)} \mathrm{d} t=(v+\eta) \int_{0}^{T} e^{\mathfrak{u}_{4}(t)} \mathrm{d} t<\left(v+\eta+\gamma e^{\mathfrak{u}_{4}\left(\theta_{3}^{*}\right)}\right) e^{\mathfrak{u}_{4}\left(\eta_{4}\right)} T .
$$

Then:

$$
e^{\mathfrak{u}_{4}\left(\eta_{4}\right)}>\frac{\gamma e^{\mathfrak{u}_{3}\left(\theta_{3}^{*}\right)}}{v+\eta+\gamma e^{\mathfrak{u}_{4}\left(\theta_{3}^{*}\right)}}:=B_{4} .
$$

Now, from Eq. (26e), there exists $\theta_{1}^{\circ} \in[0, T]$, such that:

$$
\begin{aligned}
T \eta e^{\mathfrak{u}_{1}\left(\theta_{1}^{\circ}\right)} & \leq \int_{0}^{T}\left(\beta_{2}(t) f\left(e^{\mathfrak{u}_{3}(t)}, e^{\mathfrak{u}_{7}(t)}\right)+\eta\right) e^{\mathfrak{u}_{5}(t)} \mathrm{d} t \\
& <T\left[\zeta_{2} \overline{\beta_{B}}+\eta+\eta e^{\mathfrak{u}_{1}\left(\theta_{1}^{\circ}\right)}\right] e^{\mathfrak{u}_{5}\left(\eta_{5}\right)} .
\end{aligned}
$$

Therefore, one gets:

$$
e^{\mathfrak{u}_{5}\left(\eta_{5}\right)}>\frac{\eta e^{\mathfrak{u}_{1}\left(\theta_{1}^{\circ}\right)}}{\zeta_{2} \overline{\beta_{B}}+\eta+\eta e^{\mathfrak{u}_{1}\left(\theta_{1}^{\circ}\right)}}:=B_{5} .
$$

In Eq. (26f), there exists $\theta_{2}^{\circ} \in[0, T]$, such that:

$$
\begin{aligned}
T \eta e^{\mathfrak{u}_{2}\left(\theta_{2}^{\circ}\right)} & =\eta \int_{0}^{T} e^{\mathfrak{u}_{2}(t)} \mathrm{d} t<\left(\delta+\eta+\eta e^{\mathfrak{u}_{2}\left(\theta_{2}^{\circ}\right)}\right) \int_{0}^{T} e^{\mathfrak{u}_{6}(t)} \mathrm{d} t \\
& <\left(\delta+\eta+\eta e^{\mathfrak{u}_{2}\left(\theta_{2}^{\circ}\right)}\right) e^{\mathfrak{u}_{6}\left(\eta_{6}\right)} T .
\end{aligned}
$$

It follows that:

$$
e^{\mathfrak{u}_{6}\left(\eta_{6}\right)}>\frac{\eta e^{\mathfrak{u}_{2}\left(\theta_{2}^{\circ}\right)}}{\delta+\eta+\eta e^{\mathfrak{u}_{2}\left(\theta_{2}^{\circ}\right)}}:=B_{6} .
$$

From Eq. (26g), one gets:

$$
\begin{aligned}
\eta T e^{\mathfrak{u}_{3}\left(\theta_{3}^{*}\right)} & =\eta \int_{0}^{T} e^{\mathfrak{u}_{3}(t)} \mathrm{d} t \\
& \leq(\gamma+\eta) \int_{0}^{T} e^{\mathfrak{u}_{7}(t)} \mathrm{d} t<\left(e^{\mathfrak{u}_{3}\left(\theta_{3}^{*}\right)} \eta+\gamma+\eta\right) e^{\mathfrak{u}_{7}\left(\eta_{7}\right)} T .
\end{aligned}
$$

It deduces that:

$$
e^{\mathfrak{u}_{7}\left(\eta_{7}\right)}>\frac{\eta e^{\mathfrak{u}_{3}\left(\theta_{3}^{*}\right)}}{\eta e^{\mathfrak{u}_{3}\left(\theta_{3}^{*}\right)}+\gamma+\eta}:=B_{7} .
$$

And finally from Eq. (26h), there exists $\theta_{4}^{*} \in[0, T]$, such that:

$$
\begin{aligned}
\eta T e^{\mathfrak{u}_{4}\left(\theta_{4}^{*}\right)} & =\eta \int_{0}^{T} e^{\mathfrak{u}_{4}(t)} \mathrm{d} t \\
& \leq(v+\eta) \int_{0}^{T} e^{\mathfrak{u}_{8}(t)} \mathrm{d} t<\left(e^{\mathfrak{u}_{4}\left(\theta_{4}^{*}\right)} \eta+v+\eta\right) e^{\mathfrak{u}_{8}\left(\eta_{8}\right)} T .
\end{aligned}
$$


It follows that:

$$
e^{\mathfrak{u}_{8}\left(\eta_{8}\right)}>\frac{\eta e^{\mathfrak{u}_{4}\left(\theta_{4}^{*}\right)}}{\eta e^{\mathfrak{u}_{4}\left(\theta_{4}^{*}\right)}+v+\eta}:=B_{8} .
$$

Now, from (26a)-(26d), it can been seen:

$$
\eta \int_{0}^{T} e^{\mathfrak{u}_{1}(t)} \mathrm{d} t+\eta \int_{0}^{T} e^{\mathfrak{u}_{2}(t)} \mathrm{d} t+\eta \int_{0}^{T} e^{\mathfrak{u}_{3}(t)} \mathrm{d} t+\int_{0}^{T} e^{\mathfrak{u}_{4}(t)} \mathrm{d} t=\mu T,
$$

and from (26e) to (26f), it follows that:

$$
\eta \int_{0}^{T} e^{\mathfrak{u}_{5}(t)} \mathrm{d} t+\eta \int_{0}^{T} e^{\mathfrak{u}_{6}(t)} \mathrm{d} t+\eta \int_{0}^{T} e^{\mathfrak{u}_{7}(t)} \mathrm{d} t+\int_{0}^{T} e^{\mathfrak{u}_{8}(t)} \mathrm{d} t=\mu T .
$$

Adding (35) and (36), it follows that:

$$
\sum_{i=1}^{8} \int_{0}^{T} e^{\mathfrak{u}_{\mathfrak{i}}(t)} \mathrm{d} t=\frac{2 \mu T}{\eta}
$$

Thus, we can derive:

$$
e^{\mathfrak{u}_{\mathfrak{i}}\left(\xi_{i}\right)} \leq \frac{2 \mu}{\eta}, \text { for } i=1, \ldots, 8 .
$$

Therefore, for $t \in[0, T]$, from (18) to (25),(27) to (34), and (37), one gets:

$$
\begin{aligned}
& \mathfrak{u}_{\mathfrak{i}}(t) \leq \mathfrak{u}_{\mathfrak{i}}\left(\xi_{1}\right)+\int_{\xi_{1}}^{T}\left|\dot{\mathfrak{u}}_{\mathfrak{i}}(t)\right| \mathrm{d} t \leq \mathfrak{u}_{\perp}\left(\xi_{i}\right)+\int_{0}^{T}\left|\dot{\mathfrak{u}}_{\mathfrak{i}}(t)\right| \mathrm{d} t<\ln \left(\frac{2 \mu}{\eta}\right)+k_{i}=\mathbf{H}_{\mathbf{i}}, \\
& \mathfrak{u}_{\mathfrak{i}}(t) \geq \mathfrak{u}_{\mathfrak{i}}\left(\eta_{1}\right)-\int_{0}^{T}\left|\dot{\mathfrak{u}}_{\mathfrak{i}}(t)\right| \mathrm{d} t>\ln \left(B_{i}\right)-k_{i}=\mathbf{L}_{\mathbf{i}}, i=1, \ldots, 8 .
\end{aligned}
$$

From (38), it deduces that:

$$
\max _{t \in[0, T]}\left|\mathfrak{u}_{\mathfrak{i}}(t)\right|<\max \left\{\left|\mathbf{H}_{\mathbf{i}}\right|,\left|\mathbf{L}_{\mathbf{i}}\right|\right\}:=\mathscr{R}_{i}, \text { where } i=1, \ldots, 8 .
$$

Clearly, each $\mathscr{R}_{i}(i=1, \ldots, 8)$ does not depend on $\lambda$. Let $0 \leq \mu_{0} \leq 1$, and the algebraic equations:

$$
\begin{aligned}
& \mu e^{-\mathfrak{u}_{1}}-\overline{\beta_{1}} f\left(e^{\mathfrak{u}_{3}}, e^{\mathfrak{u}_{7}}\right)+\mu_{0} v e^{\mathfrak{u}_{4}-\mathfrak{u}_{1}}-\eta=0, \\
& \overline{\beta_{1}} e^{\mathfrak{u}_{1}-\mathfrak{u}_{2}} f\left(e^{\mathfrak{u}_{3}}, e^{\mathfrak{u}_{7}}\right)-\delta-\eta=0, \\
& \delta e^{\mathfrak{u}_{2}-\mathfrak{u}_{3}}-\gamma-\eta=0, \\
& \gamma e^{\mathfrak{u}_{3}-\mathfrak{u}_{4}}-v-\eta=0, \\
& \eta e^{\mathfrak{u}_{1}-\mathfrak{u}_{5}}-\overline{\beta_{2}} f\left(e^{\mathfrak{u}_{3}}, e^{\mathfrak{u}_{7}}\right)+\mu_{0} v e^{\mathfrak{u}_{8}-\mathfrak{u}_{5}}-\eta=0, \\
& \mu_{0} \eta e^{\mathfrak{u}_{2}-\mathfrak{u}_{6}}+\overline{\beta_{2}} e^{\mathfrak{u}_{5}-\mathfrak{u}_{6}} f\left(e^{\mathfrak{u}_{3}}, e^{\mathfrak{u}_{7}}\right)-\delta-\eta=0, \\
& \mu_{0} \eta e^{\mathfrak{u}_{3}-\mathfrak{u}_{7}}+\delta e^{\mathfrak{u}_{6}-\mathfrak{u}_{7}}-\gamma-\eta=0, \\
& \mu_{0} \eta e^{\mathfrak{u}_{4}-\mathfrak{u}_{8}}+\gamma e^{\mathfrak{u}_{7}-\mathfrak{u}_{8}}-v-\eta=0,
\end{aligned}
$$

where $\left(\mathfrak{u}_{1}, \ldots, \mathfrak{u}_{8}\right)^{T}$ in $\mathbb{R}^{8}$. Now, from (40a) to (40h), we can deduce:

$$
\begin{aligned}
& \mu+v e^{\mathfrak{u}_{4}} \geq(\delta+\eta) e^{\mathfrak{u}_{2}}, \\
& \delta e^{\mathfrak{u}_{2}}=(\gamma+\eta) e^{\mathfrak{u}_{3}},
\end{aligned}
$$




$$
\begin{aligned}
& \gamma e^{\mathfrak{u}_{3}}=(v+\eta) e^{\mathfrak{u}_{4}}, \\
& \eta e^{\mathfrak{u}_{1}}+\eta e^{\mathfrak{u}_{2}}+v e^{\mathfrak{u}_{8}} \geq(\delta+\eta) e^{\mathfrak{u}_{6}}, \\
& \eta e^{\mathfrak{u}_{3}}+\delta e^{\mathfrak{u}_{6}} \geq(\gamma+\eta) e^{\mathfrak{u}_{7}}, \\
& \eta e^{\mathfrak{u}_{4}}+\gamma e^{\mathfrak{u}_{7}} \geq(\nu+\eta) e^{\mathfrak{u}_{8}} .
\end{aligned}
$$

Thus, summing from (41a) to (41c), one gets that:

$$
\sum_{i=2}^{4} e^{\mathfrak{u}_{\mathfrak{i}}}<\frac{\mu}{\eta} .
$$

Moreover, from Eq. (40a), it follows that:

$$
\mu+v e^{\mathfrak{u}_{4}}>\left(\overline{\beta_{1}} f\left(e^{\mathfrak{u}_{3}}, e^{\mathfrak{u}_{3}}\right)+\eta\right) e^{\mathfrak{u}_{1}}>\eta e^{\mathfrak{u}_{1}},
$$

and using (42), we obtain:

$$
\frac{\mu(\nu+\eta)}{\eta^{2}}>e^{\mathfrak{u}_{\perp}} .
$$

Choosing,

$$
C_{1}:=\frac{\mu(v+\eta)}{\eta^{2}},
$$

we can see that:

$$
\sum_{i=1}^{4} e^{\mathfrak{u}_{\mathfrak{i}}}<\frac{\mu}{\eta}+C_{1}:=M_{0} .
$$

Now, from (41d) to (41f), one gets that:

$$
\sum_{i=6}^{8} e^{\mathfrak{u}_{\mathfrak{i}}} \leq M_{0} .
$$

Using Eqs. (40e) and (43), we obtain that:

$$
e^{u_{5}}<\frac{\mu(v+\eta)+v \eta M_{0}}{\eta^{2}}:=C_{2} .
$$

Therefore, $e^{u_{i}}<2 M_{0}+C_{2}:=M_{M}$, for $i=1, \ldots, 8$. Next, Eq. (40a) implies that:

$$
\mu<\overline{\beta_{1}} e^{\mathfrak{u}_{1}} f\left(e^{\mathfrak{u}_{3}}, e^{\mathfrak{u}_{3}}\right)+\eta e^{\mathfrak{u}_{1}}, \text { thus } e^{\mathfrak{u}_{1}}>\frac{\mu}{\eta+\overline{\beta_{1}} \zeta_{2}+\mu}:=m_{1},
$$

and using (48), we derive that:

$$
\mu+v e^{\mathfrak{u}_{4}} \geq \mu+\mu_{0} v e^{\mathfrak{u}_{4}}>\eta e^{\mathfrak{u}_{1}}+\overline{\beta_{1}} e^{\mathfrak{u}_{1}} f\left(e^{\mathfrak{u}_{3}}, e^{\mathfrak{u}_{3}}\right)>\eta e^{\mathfrak{u}_{1}}>\frac{\eta \mu}{\eta+\overline{\beta_{1}} \zeta_{2}+\mu} .
$$

Thus:

$$
\mu+v e^{\mathfrak{u}_{4}}>\frac{\eta \mu}{\eta+\overline{\beta_{1}} \zeta_{2}+\mu}
$$

Choosing

$$
e^{\mathfrak{u}_{4}}>\frac{\eta \mu}{v \eta+v \overline{\beta_{1}} \zeta_{2}+v \mu}:=m_{4},
$$


we can see that (49) is holds. Now, from (41c) and (41b), it follows that:

$$
e^{\mathfrak{u}_{3}}>\frac{(v+\eta) m_{4}}{\gamma}:=m_{3},
$$

and

$$
e^{\mathfrak{u}_{2}}>\frac{(\gamma+\eta) m_{3}}{\delta}:=m_{2}
$$

Next, from (40e) and (40f):

$$
\eta m_{1}<\eta e^{\mathfrak{u}_{1}} \leq \eta e^{\mathfrak{u}_{1}}+\mu_{0} v e^{\mathfrak{u}_{8}}=\left(\eta+\overline{\beta_{2}} f\left(e^{\mathfrak{u}_{3}}, e^{\mathfrak{u}_{3}}\right)\right) e^{\mathfrak{u}_{5}} ;
$$

that is:

$$
e^{\mathfrak{u}_{5}}>\frac{\eta m_{1}}{\eta m_{1}+\overline{\beta_{2}} \zeta_{2}+\eta}:=m_{5},
$$

and

$$
\eta m_{1}<(\delta+\eta) e^{\mathfrak{u}_{6}}+\eta e^{\mathfrak{u}_{5}}
$$

We can see that $e^{\mathfrak{u}_{6}}>\frac{m_{1} \eta}{(\delta+\eta)}:=m_{6}$, holds with (54). Finally, from (40g) and (40h), one gets that:

$$
\begin{aligned}
& e^{\mathfrak{u}_{7}}>\frac{\delta e^{\mathfrak{u}_{6}}}{\gamma+\eta}>\frac{\delta m_{6}}{\gamma+\eta+\delta m_{6}}:=m_{7}, \text { and } \\
& e^{\mathfrak{u}_{8}}>\frac{\gamma e^{\mathfrak{u}_{7}}}{v+\eta}>\frac{\gamma m_{7}}{v+\eta+\gamma m_{7}}:=m_{8} .
\end{aligned}
$$

If we select $\mathscr{R}=\max _{i \in\{1, \ldots, 8\}}\left\{\left|\ln \left(M_{M}\right)\right|,\left|\ln \left(m_{i}\right)\right|\right\}$, then:

$$
\sum_{i=1}^{8}\left|\mathfrak{u}_{\mathfrak{i}}\right|<8 \mathscr{R}:=\mathscr{R}_{0}
$$

Thus, with $\mathscr{R}_{T}=\sum_{i=0}^{8} \mathscr{R}_{i}+\mathscr{R}_{0}$, we define:

$$
\Omega_{0}=\left\{\mathfrak{u}(t) \in \mathscr{X}:\|\mathfrak{u}\|<\mathscr{R}_{T}\right\} .
$$

Therefore, $\mathscr{L} \mathfrak{u} \neq \lambda \mathscr{N} \mathfrak{u}$, with $\lambda \in(0,1)$, and $\mathfrak{u} \in \partial \Omega_{0} \cap$ Dom $\mathscr{L}$, and thus, $\Omega_{0}$ holds condition (1) of Theorem 1.

For $\mathfrak{u}=\left(\mathfrak{u}_{1}, \ldots, \mathfrak{u}_{8}\right)^{T} \in \partial \Omega_{0} \cap \operatorname{Ker} \mathscr{L}=\partial \Omega_{0} \cap \mathbb{R}^{8}, \mathfrak{u}$ is a constant vector in $\mathbb{R}^{8}$ with $\|\mathfrak{u}\|=\mathscr{R}_{T}$. If $\mathscr{Q} \mathscr{N} \mathfrak{u}=0$, then $\left(\mathfrak{u}_{1}, \ldots, \mathfrak{u}_{8}\right)^{T}$ is a constant solution of system $(40 \mathrm{a}-40 \mathrm{~h})$ with $\mu_{0}=1$. From (57), we have that $\|\mathfrak{u}\|<\mathscr{R}_{0}$ which is contradictory to $\|\mathfrak{u}\|=\mathscr{R}_{T}$. It follows that for each $\mathfrak{u} \in \partial \Omega_{0} \cap \operatorname{Ker} \mathscr{L}, \mathscr{Q} \mathscr{N} \mathfrak{u} \neq 0$. This shows that condition (2) of Theorem 1 is satisfied.

To verify the condition (3) of the Continuation Theorem, we define $\phi: \operatorname{Dom} \mathscr{L} \cap \operatorname{Ker} \mathscr{L} \times[0,1] \longrightarrow \mathscr{X}$ by: 


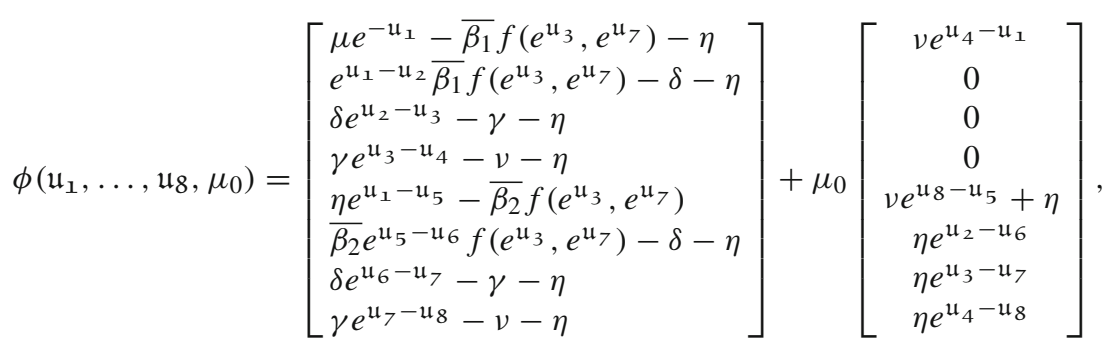

where $\mu_{0} \in[0,1]$. If $\mathfrak{u}=\left(\mathfrak{u}_{1}, \ldots, \mathfrak{u}_{8}\right)^{T} \in \partial \Omega_{0} \cap \operatorname{Ker} \mathscr{L}=\partial \Omega_{0} \cap \mathbb{R}^{8}$, then $\mathfrak{u}$ is constant, such that $\mathfrak{u} \in \mathbb{R}^{8},\|\mathfrak{u}\|=\mathscr{R}_{T}$ and $\phi\left(\mathfrak{u}_{1}, \ldots, \mathfrak{u}_{8}, \mu_{0}\right) \neq 0$. Therefore:

$$
\begin{aligned}
\operatorname{deg} & \left(\mathscr{J} \mathscr{Q} \mathscr{N}\left(\left(\mathfrak{u}_{1}, \ldots, \mathfrak{u}_{8}\right)^{T}\right), \partial \Omega_{0} \cap \operatorname{Ker} \mathscr{L},(0,0,0,0,0,0,0,0)^{T}\right) \\
= & \operatorname{deg}\left(\phi\left(\mathfrak{u}_{1}, \ldots, \mathfrak{u}_{8}, 1\right), \Omega_{0} \cap \operatorname{Ker} \mathscr{L},(0,0,0,0,0,0,0,0)^{T}\right) \\
= & \operatorname{deg}\left(\phi\left(\mathfrak{u}_{1}, \ldots, \mathfrak{u}_{8}, 0\right), \Omega_{0} \cap \operatorname{Ker} \mathscr{L},(0,0,0,0,0,0,0,0)^{T}\right) \\
= & \operatorname{deg}\left\{\left(\mu e^{-\mathfrak{u}_{1}}-\overline{\beta_{1}} f\left(e^{\mathfrak{u}_{3}}, e^{\mathfrak{u}_{7}}\right)-\eta, e^{\mathfrak{u}_{1}-\mathfrak{u}_{2}} \overline{\beta_{1}} f\left(e^{\mathfrak{u}_{3}}, e^{\mathfrak{u}_{7}}\right)\right.\right. \\
& -\delta-\eta, \delta e^{\mathfrak{u}_{2}-\mathfrak{u}_{3}}-\gamma-\eta, \\
& \gamma e^{\mathfrak{u}_{3}-\mathfrak{u}_{4}}-v-\eta, \eta e^{\mathfrak{u}_{1}-\mathfrak{u}_{5}}-\overline{\beta_{2}} f\left(e^{\mathfrak{u}_{3}}, e^{\mathfrak{u}_{7}}\right), \overline{\beta_{2}} e^{\mathfrak{u}_{5}-\mathfrak{u}_{6}} f\left(e^{\mathfrak{u}_{3}}, e^{\mathfrak{u}_{3}}\right)-\delta-\eta, \\
& \left.\left.\delta e^{\mathfrak{u}_{6}-\mathfrak{u}_{7}}-\gamma-\eta, \gamma e^{\mathfrak{u}_{7}-\mathfrak{u}_{8}}-v-\eta\right)^{T}, \Omega_{0} \cap \operatorname{Ker} \mathscr{L},(0,0,0,0,0,0,0,0)^{T}\right\} .
\end{aligned}
$$

Using Proposition (1), we obtain that:

$$
\begin{aligned}
& \operatorname{deg}\left(\mathscr{J} \mathscr{Q} \mathscr{N}\left(\left(\mathfrak{u}_{1}, \ldots, \mathfrak{u}_{8}\right)^{T}\right), \partial \Omega_{0} \cap \operatorname{Ker} \mathscr{L},(0,0,0,0,0,0,0,0)^{T}\right)
\end{aligned}
$$

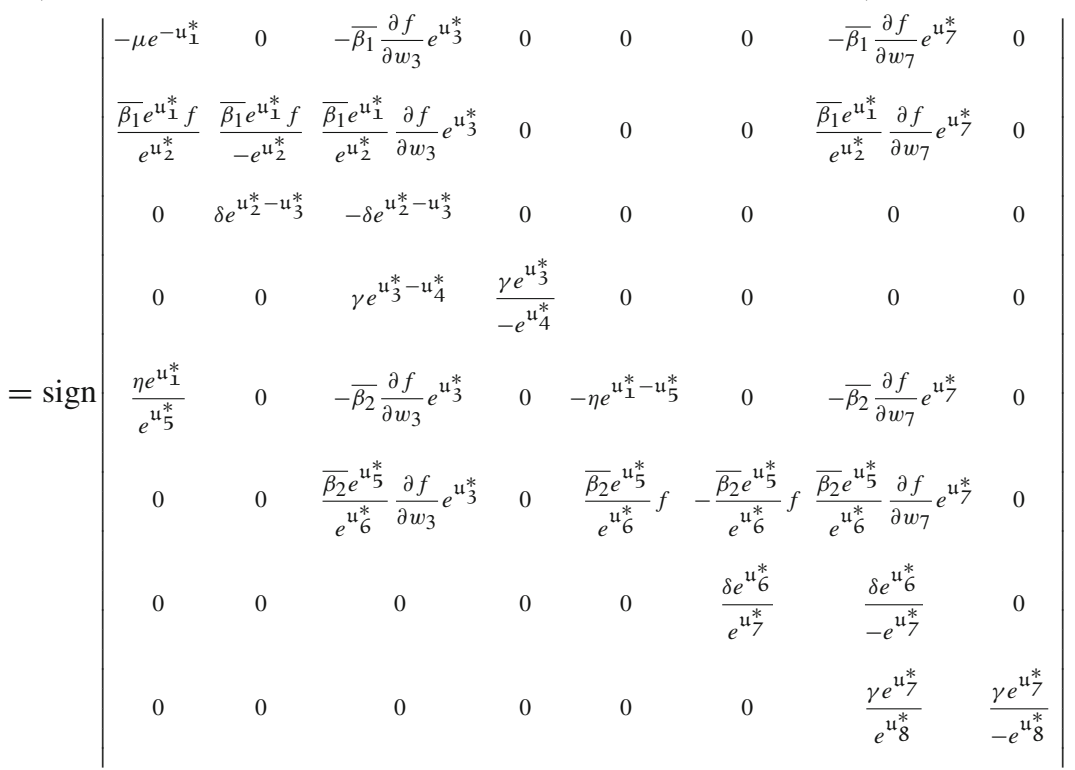




$$
\begin{aligned}
= & \operatorname{sign}\left(\overline { \beta _ { 2 } \beta _ { 1 } } \gamma ^ { 2 } \delta ^ { 2 } e ^ { 8 \mathfrak { u } _ { \perp } ^ { * } } \left\{e^{-\mathfrak{u}_{\perp}^{*}} f\left[\eta \overline{\beta_{1}} \frac{\partial f}{\partial w_{3}} e^{\mathfrak{u}_{3}^{*}}+\eta \overline{\beta_{1}} \frac{\partial f}{\partial w_{7}} e^{\mathfrak{u}_{7}^{*}}+\mu \overline{\beta_{2}} \frac{\partial f}{\partial w_{7}} e^{\mathfrak{u}_{7}^{*}}\right]\right.\right. \\
& \left.\left.-\mu \eta\left[\frac{\partial f}{\partial w_{3}} e^{\mathfrak{u}_{3}^{*}}+\frac{\partial f}{\partial w_{7}} e^{\mathfrak{u}_{7}^{*}}-f\right]\right\}\right) \neq 0 .
\end{aligned}
$$

Consequently, by Theorem 1, the system (4) has at least one periodic solution in $\operatorname{Dom} \mathscr{L} \cap \overline{\Omega_{0}}$.

Remark $2 f:=f\left(e^{\mathfrak{u}_{3}^{*}}, e^{\mathfrak{u}_{7}^{*}}\right)$ and $\frac{\partial f}{\partial w_{3}}:=\frac{\partial f\left(e^{\mathfrak{u}_{3}^{*}}, e^{\mathfrak{u}_{7}^{*}}\right)}{\partial w_{3}}, \frac{\partial f}{\partial w_{7}}:=\frac{\partial f\left(e^{\mathfrak{u}_{3}^{*}}, e^{\mathfrak{u}_{7}^{*}}\right.}{\partial w_{7}}$, and $e^{\mathfrak{u}_{i}^{*}}$ are the solutions of system given in Proposition 1.

\section{Global attractivity}

Next, we analyze the conditions for which the solution of system (4) is globally attractive.

Definition 1 System (4) is said to be globally attractive if there exists a positive $T$-periodic solution $\left(w_{1}(t), \ldots, w_{8}(t)\right)$, such that for any $\left(y_{1}(t), \ldots, y_{8}(t)\right)$ arbitrary positive solution of system (4), it holds:

$$
\lim _{t \rightarrow \infty} \sum_{i=1}^{8}\left|w_{i}(t)-y_{i}(t)\right|=0,
$$

and the solution $\left(w_{1}(t), \ldots, w_{8}(t)\right)$ is called globally attractive, see (Guo and Chen 2011; Yan and Sugie 2020; Zhu and Wang 2011).

Without loss of generality, we will use the following space:

$$
\mathscr{X}=\left\{w(t)=\left(w_{1}(t), \ldots, w_{8}(t)\right)^{T} \in C\left(\mathbb{R}, \mathbb{R}^{8}\right) / w(T+t)=w(t)\right\}
$$

with norm

$$
\|w(t)\|=\sum_{i=1}^{8} \frac{\left|w_{i}(t)\right|}{d_{i}} .
$$

From now on, we use the notation:

$$
\begin{aligned}
-p & :=\eta\left[\frac{d_{1}}{d_{5}}+\frac{d_{2}}{d_{6}}+\frac{d_{3}}{d_{7}}+\frac{d_{4}}{d_{8}}\right]+v\left[\frac{d_{4}}{d_{1}}+\frac{d_{8}}{d_{5}}\right]+\delta\left[\frac{d_{2}}{d_{3}}+\frac{d_{6}}{d_{7}}\right]+\gamma\left[\frac{d_{3}}{d_{4}}+\frac{d_{7}}{d_{8}}\right]-\eta, \\
q & :=\beta_{1}^{u} K\left[\left(1+\alpha+\frac{d_{1}}{d_{2}}+\alpha \frac{d_{5}}{d_{6}}\right)\left(d_{3}+d_{7}\right)\right],
\end{aligned}
$$

with $K=\max _{t \in[0, T]}\left\{\frac{\partial f\left(w_{3}, w_{7}\right)}{\partial w_{3}}, \frac{\partial f\left(w_{3}, w_{7}\right)}{\partial w_{7}}\right\}$, and we also assume that:

$$
\begin{aligned}
& {\left[\mathrm{H}_{6}\right] I_{1}=\frac{p+\sqrt{p^{2}-4 q \frac{\mu}{d_{1}}}}{2 q}>0,} \\
& {\left[\mathrm{H}_{7}\right] I_{2}=\frac{p-\sqrt{p^{2}-4 q \frac{\mu}{d_{1}}}}{2 q}, \text { where } p^{2}-4 q \frac{\mu}{d_{1}}>0 .}
\end{aligned}
$$


Theorem 3 If conditions $\left[\mathrm{H}_{1}\right]-\left[\mathrm{H}_{7}\right]$ hold, then there exists a positive constant $\mathfrak{B} \in\left[I_{1}, I_{2}\right]$, such that for all $w(t)$ solution of (4), it holds:

$$
\|w(t)\|<\mathfrak{B} \text { for all } t \geq 0 .
$$

Proof If (59) is not true, then there exists $t_{0}>0$, such that:

$$
\left\|w\left(t_{0}\right)\right\|=\mathfrak{B} \text { and }\|w(t)\|<\mathfrak{B}, t \in\left[0, t_{0}\right) .
$$

Now, for $0 \leq t<t_{0}$ and from (4), we have that:

$$
\begin{aligned}
\left\|w\left(t_{0}\right)\right\| \leq & \|w(0)\| e^{-\eta t_{0}} \\
& +\int_{0}^{t_{0}} e^{-\eta\left(t_{0}-s\right)}\left\{\frac{\mu}{d_{1}}+\beta_{1}^{u} K \mathfrak{B}^{2}\left(d_{3}+d_{7}\right)+\frac{\nu d_{4} \mathfrak{B}}{d_{1}}\right\} \mathrm{d} s \\
& +\int_{0}^{t_{0}} e^{-(\delta+\eta)\left(t_{0}-s\right)}\left\{\beta_{1}^{u} \frac{d_{1}}{d_{2}} \zeta_{2} \mathfrak{B}\right\} \mathrm{d} s+\int_{0}^{t_{0}} e^{-(\gamma+\eta)\left(t_{0}-s\right)}\left\{\delta d_{3}^{-1} d_{2} \mathfrak{B}\right\} \mathrm{d} s \\
& +\int_{0}^{t_{0}} e^{-(\nu+\eta)\left(t_{0}-s\right)}\left\{\gamma d_{3} d_{4}^{-1} \mathfrak{B}\right\} \mathrm{d} s \\
& +\int_{0}^{t_{0}} e^{-\eta\left(t_{0}-s\right)}\left\{\left(\frac{\eta d_{1}}{d_{5}}+\frac{\nu d_{8}}{d_{5}}\right) \mathfrak{B}+\beta_{1}^{u} \alpha K \mathfrak{B}^{2}\left(d_{3}+d_{7}\right)\right\} \mathrm{d} s \\
& +\int_{0}^{t_{0}} e^{-(\delta+\eta)\left(t_{0}-s\right)}\left\{\frac{\eta d_{2}}{d_{6}} \mathfrak{B}+\beta_{1}^{u} \alpha K \mathfrak{B}^{2}\left(d_{3}+d_{7}\right) \frac{d_{5}}{d_{6}}\right\} \mathrm{d} s \\
& +\int_{0}^{t_{0}} e^{-(\gamma+\eta)\left(t_{0}-s\right)}\left\{\left(\frac{\eta d_{3}}{d_{7}}+\frac{\delta d_{6}}{d_{7}}\right) \mathfrak{B}\right\} \mathrm{d} s \\
& +\int_{0}^{t_{0}} e^{-(\nu+\eta)\left(t_{0}-s\right)}\left\{\left(\frac{\eta d_{4}}{d_{8}}+\frac{\gamma d_{7}}{d_{8}}\right) \mathfrak{B}\right\} \mathrm{d} s .
\end{aligned}
$$

Thus:

$$
\begin{aligned}
\mathfrak{B}= & \left\|w\left(t_{0}\right)\right\|<\mathfrak{B} e^{-\eta t_{0}}+\frac{\beta_{1}^{u} K \mathfrak{B}^{2}}{\eta}\left[\left(1+\alpha+\frac{d_{1}}{d_{2}}+\alpha \frac{d_{5}}{d_{6}}\right)\left(d_{3}+d_{7}\right)\right] \\
& +\frac{\mathfrak{B}}{\eta}\left[\eta\left[\frac{d_{1}}{d_{5}}+\frac{d_{2}}{d_{6}}+\frac{d_{3}}{d_{7}}+\frac{d_{4}}{d_{8}}\right]+v\left[\frac{d_{4}}{d_{1}}+\frac{d_{8}}{d_{5}}\right]+\delta\left[\frac{d_{2}}{d_{3}}+\frac{d_{6}}{d_{7}}\right]\right. \\
& \left.+\gamma\left[\frac{d_{3}}{d_{4}}+\frac{d_{7}}{d_{8}}\right]+\beta_{1}^{u} \frac{d_{1}}{d_{2}} \zeta_{2}\right]\left(1-e^{-\eta t_{0}}\right)+\frac{\mu}{\eta d_{1}} .
\end{aligned}
$$

Therefore:

$$
\begin{aligned}
0< & \frac{\beta_{1}^{u} K \mathfrak{B}^{2}}{\eta}\left[\left(1+\alpha+\frac{d_{1}}{d_{2}}+\alpha \frac{d_{5}}{d_{6}}\right)\left(d_{3}+d_{7}\right)\right]+\frac{\mu}{\eta d_{1}} \\
& +\frac{\mathfrak{B}}{\eta}\left[\eta\left[\frac{d_{1}}{d_{5}}+\frac{d_{2}}{d_{6}}+\frac{d_{3}}{d_{7}}+\frac{d_{4}}{d_{8}}\right]+v\left[\frac{d_{4}}{d_{1}}+\frac{d_{8}}{d_{5}}\right]+\delta\left[\frac{d_{2}}{d_{3}}+\frac{d_{6}}{d_{7}}\right]+\gamma\left[\frac{d_{3}}{d_{4}}+\frac{d_{7}}{d_{8}}\right]\right] .
\end{aligned}
$$

Accordingly:

$$
\left\{\left[\frac{d_{1}}{d_{5}}+\frac{d_{2}}{d_{6}}+\frac{d_{3}}{d_{7}}+\frac{d_{4}}{d_{8}}\right]+v\left[\frac{d_{4}}{d_{1}}+\frac{d_{8}}{d_{5}}\right]+\delta\left[\frac{d_{2}}{d_{3}}+\frac{d_{6}}{d_{7}}\right]+\gamma\left[\frac{d_{3}}{d_{4}}+\frac{d_{7}}{d_{8}}\right]\right\}^{2}<\frac{4 \mu q}{d_{1}},
$$

which contradicts $\mathrm{H}_{7}$. Thus, the proof is completed.

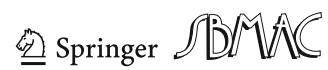


Now, let $\left(w_{1}(t), \ldots, w_{8}(t)\right)$ and $\left(y_{1}(t), \ldots, y_{8}(t)\right)$ be two positive $T$-periodic solutions of system (4), and define:

$$
z_{i}(t)=\left(w_{i}(t)-y_{i}(t)\right)
$$

then, system (4) is written as:

$$
\begin{aligned}
& \dot{z_{1}}(t)+\left(\eta+\beta_{1}(t) f\left(w_{3}(t), w_{7}(t)\right)\right) z_{1}(t)=-\beta_{1}(t) y_{1}(t) f\left(w_{3}(t), w_{7}(t)\right) \\
& \quad+\beta_{1}(t) y_{1}(t) f\left(y_{3}(t), y_{7}(t)\right)+v z_{4}(t), \\
& \dot{z_{2}}(t)+(\delta+\eta) z_{2}(t)=\beta_{1}(t) z_{1}(t) f\left(w_{3}(t), w_{7}(t)\right) \\
& \quad+\beta_{1}(t) y_{1}(t) f\left(w_{3}(t), w_{7}(t)\right)-\beta_{1}(t) y_{1}(t) f\left(y_{3}(t), y_{7}(t)\right), \\
& \dot{z_{3}}(t)+(\gamma+\eta) z_{3}(t)=\delta z_{2}(t), \\
& \dot{z_{4}}(t)+(v+\eta) z_{4}(t)=\gamma z_{3}(t), \\
& \quad \dot{z_{5}}(t)+\left(\eta+\beta_{2}(t) f\left(w_{3}(t), w_{7}(t)\right)\right) z_{5}(t)=-\beta_{2}(t) y_{5}(t) f\left(w_{3}(t), w_{7}(t)\right) \\
& \quad+\beta_{2}(t) y_{5}(t) f\left(y_{3}(t), y_{7}(t)\right)+v z_{8}(t)+\eta z_{1}(t), \\
& \quad \dot{z_{6}}(t)+(\delta+\eta) z_{6}(t)=\beta_{2}(t) y_{5}(t) f\left(w_{3}(t), w_{7}(t)\right) \\
& \quad-\beta_{2}(t) y_{5}(t) f\left(y_{3}(t), y_{7}(t)\right)+\eta z_{2}(t), \\
& \dot{z_{7}}(t)+(\gamma+\eta) z_{7}(t)=\eta z_{3}(t)+\delta z_{6}(t), \\
& \dot{z_{8}}(t)+(v+\eta) z_{8}(t)=\eta z_{4}(t)+\gamma z_{7}(t) .
\end{aligned}
$$

Thus, the solution $\left(w_{1}(t), \ldots, w_{8}(t)\right)$ is globally attractive to the system (4) if and only if the zero solution of system (62) is globally attractive.

Theorem 4 If $\left[\mathrm{H}_{1}\right]-\left[\mathrm{H}_{7}\right]$ hold, then the solution $\left(0_{1}(t), \ldots, 0_{8}(t)\right)$ to the system $(62)$ is global attractive.

Proof From Theorem 3, we can see that there exists a non-negative constant $\rho$, such that:

$$
\limsup _{t \longrightarrow \infty}\|z(t)\|=\rho .
$$

Thus, for $\epsilon>0$ small enough, there is a $t_{1}>0$, such that:

$$
\|z(t)\|=(1+\epsilon) \rho, \text { for } t \geq t_{1} .
$$

From system (62), when $t \geq t_{1}$, one gets:

$$
\begin{aligned}
& \quad \dot{z_{1}}(t)+\left(\eta+\beta_{1}(t) f\left(w_{3}(t), w_{7}(t)\right)\right) z_{1}(t) \leq \beta_{1}^{u} y_{1}(t)\left|z_{3}(t)\right| K+\beta_{1}^{u} y_{1}(t)\left|z_{7}(t)\right| K \\
& \quad+v\left|z_{4}(t)\right|, \\
& \dot{z_{2}}(t)+(\delta+\eta) z_{2}(t) \leq \beta_{1}^{u}\left|z_{1}(t)\right| \zeta_{2}+\beta_{1}^{u} y_{1}(t)\left|z_{3}(t)\right| K+\beta_{1}^{u} y_{1}(t)\left|z_{7}(t)\right| K, \\
& \dot{z_{3}}(t)+(\gamma+\eta) z_{3}(t) \leq \delta\left|z_{2}(t)\right|, \\
& \dot{z_{4}}(t)+(v+\eta) z_{4}(t) \leq \gamma\left|z_{3}(t)\right|, \\
& \dot{z_{5}}(t)+\left(\eta+\beta_{2}(t) f\left(w_{3}(t), w_{7}(t)\right)\right) z_{5}(t) \leq \beta_{2}^{u} y_{5}(t)\left|z_{3}(t)\right| K+\beta_{2}^{u} y_{5}(t)\left|z_{7}(t)\right| K \\
& \quad+v\left|z_{8}(t)\right|+\eta\left|z_{1}(t)\right|, \\
& \dot{z_{6}}(t)+(\delta+\eta) z_{6}(t) \leq \beta_{2}^{u} y_{5}(t) K\left|z_{3}(t)\right|+\beta_{2}^{u} y_{5}(t) K\left|z_{7}(t)\right|+\eta\left|z_{2}(t)\right|, \\
& \dot{z_{7}}(t)+(\gamma+\eta) z_{7}(t) \leq \eta\left|z_{3}(t)\right|+\delta\left|z_{6}(t)\right|, \\
& \dot{z_{8}}(t)+(v+\eta) z_{8}(t) \leq \eta\left|z_{4}(t)\right|+\gamma\left|z_{7}(t)\right| .
\end{aligned}
$$


Solving the system (65), one obtains that:

$$
\begin{aligned}
\|z(t)\| & \leq\|z(0)\| e^{-\eta t}+\int_{0}^{t_{0}} e^{-\eta(t-s)}\left\{q \mathfrak{B}+\left(\eta+\beta_{1}^{u} \frac{d_{1}}{d_{2}} \zeta_{2}-p\right)\right\} \frac{(1+\epsilon) \rho}{\eta} \mathrm{d} s \\
& =\|z(0)\| e^{-\eta t}+\left\{q \mathfrak{B}+\left(\eta+\beta_{1}^{u} \frac{d_{1}}{d_{2}} \zeta_{2}-p\right)\right\} \frac{(1+\epsilon) \rho}{\eta}\left(1-e^{-\eta t}\right) .
\end{aligned}
$$

When $t \longrightarrow \infty$ and $\epsilon \longrightarrow 0$, it follows that:

$$
\rho \leq\left\{q \mathfrak{B}+\left(\eta+\beta_{1}^{u} \frac{d_{1}}{d_{2}} \zeta_{2}-p\right)\right\} \frac{\rho}{\eta} .
$$

Now, if $\rho \neq 0$, then:

$$
\eta \leq\left\{q \mathfrak{B}+\left(\eta+\beta_{1}^{u} \frac{d_{1}}{d_{2}} \zeta_{2}-p\right)\right\},
$$

which means that:

$$
0 \leq q \mathfrak{B}^{2}+\left(\beta_{1}^{u} \frac{d_{1}}{d_{2}} \zeta_{2}-p\right) \mathfrak{B} .
$$

On the other hand, from Theorem 3 for all $t>0$, we have that:

$$
\begin{aligned}
\|z(t)\| & <\mathfrak{B} e^{-\eta t}+\frac{\mathfrak{B}^{2}}{\eta} q+\frac{\mathfrak{B}}{\eta}\left(-p+\eta+\beta_{1}^{u} \frac{d_{1}}{d_{2}} \zeta_{2}\right)\left(1-e^{-\eta t}\right)+\frac{\mu}{\eta d_{1}} \\
& <\mathfrak{B} e^{-\eta t}+\frac{\mathfrak{B}^{2}}{\eta} q+\frac{\mathfrak{B}}{\eta}\left(-p+\eta+\beta_{1}^{u} \frac{d_{1}}{d_{2}} \zeta_{2}\right)+\frac{\mu}{\eta d_{1}}<\mathfrak{B} .
\end{aligned}
$$

Thus:

$$
q \mathfrak{B}^{2}+\mathfrak{B}\left(-p+\beta_{1}^{u} \frac{d_{1}}{d_{2}} \zeta_{2}\right)+\frac{\mu}{d_{1}}<0 .
$$

Therefore, $\rho=0$. This implies that $\lim \sup _{t \rightarrow \infty}\|z(t)\|=0$. Then, $\lim _{t \rightarrow \infty} \mid w_{i}(t)-$ $y_{i}(t) \mid=0$, for $i=1, \ldots, 8$. This completes the proof of Theorem 4, which implies that system (4) is globally attractive.

\section{Numerical simulations}

To exemplify the behavior of the analytical solution of system (4) and corroborate the previous theoretical results, we perform numerical simulations using the Matlab routine ode45 for solving systems of differential equations. We use a particular set of biologically feasible parameter values for the RSV transmission for children. These values are given in Table 1, and were taken from Hogan et al. (2016b). Parameters $\mu, b_{0}, b_{1}, \phi$ correspond, respectively, to the birth (death) rate, average of the transmission rate, and amplitude of the seasonal fluctuation, and $0 \leq \varphi \leq 1$ is the phase angle normalized. The parameters $\gamma$ and $v$ are the rates of immunity lost and recover of the transmission of virus RSV, respectively.

We use a canonical periodic positive solution [see Coleman et al. (1979)] to compare with solutions of the system (3), and thus corroborate the previous theoretical results obtained in the previous sections. We use two different initial conditions to generate two solutions that approach to the aforementioned canonical periodic solution. The initial conditions for the canonical and the other two solutions are presented in Table 2. 
Table 1 The values $\frac{1}{\mu}, \frac{1}{v}, \frac{1}{\gamma}$, are expressed in rates per days

\begin{tabular}{lllllllll}
\hline Country & $\mu$ & $v$ & $\gamma$ & $b_{0}$ & $b_{1}$ & $\phi$ & $\alpha$ & $\eta$ \\
\hline Australia & 0.0135 & $1 / 230$ & $1 / 4$ & 3215 & 0.522 & 0 & 0.228 & 1
\end{tabular}

The phase angle in years is denoted by $\phi$

\begin{tabular}{lll}
\hline Canonical solution & Positive solution & Positive solution \\
\hline 0.000614 & 0.00065 & 0.00055 \\
0.010419222 & 0.0106 & 0.0108 \\
0.002345668 & 0.002345 & 0.0023465 \\
0.000121341 & 0.00012152 & 0.00012154 \\
0.000155855 & 0.000144 & 0.000149 \\
0.00894556 & 0.00901 & 0.00902 \\
0.004126885 & 0.0041432 & 0.0041433 \\
0.00026976345 & 0.000269764 & 0.00026975 \\
\hline
\end{tabular}

Table 2 The initial conditions for the numerical simulations

In Fig. 1, it can be observed three numerical solutions of the system (4), where the canonical periodic positive solution is the reference to compare the attractive behavior that it produces on the two other positive solutions with different initial conditions. Moreover, all the numerical solutions are positive and have a periodic behavior (using Definition 1). This numerical result has a good agreement with our previous theoretical results. Furthermore, in Fig. 1, it can be observed the numerical solution of the proportion of susceptible children group 1 $\left(S_{1}\right)$, exposed children group $1\left(E_{1}\right)$, infected children group $1\left(I_{1}\right)$, recovered children group $1\left(R_{1}\right)$, susceptible children group $2\left(S_{2}\right)$, exposed children group $2\left(E_{2}\right)$, infected children group $2\left(I_{2}\right)$, and recovered children group $2\left(R_{2}\right)$, for the system $(4)$ using parameter values corresponding to Australia. As it can be seen, all the numerical solutions are positive and have a periodic behavior, and these results have a good agreement with our theoretical results. From a biological point of view, the two age groups can be applied to different diseases, where the individuals in each age group have different characteristics in regard to the disease.

\section{Conclusions}

In this article, we studied the dynamics of a proposed age-structured population mathematical model driven by a seasonal forcing function. We proposed a generalized force of infection function to study different potential epidemic outcomes. We established sufficient criteria for the existence of positive periodic solutions in an age-structured ordinary differential equation SEIR model with a force of infection $\beta_{i}(t) f\left(I_{1}(t), I_{2}(t)\right)$. We defined a convenient norm function, to provide sufficient conditions that assure the global attractivity of the positive periodic solution.

The proposed model includes two age groups that can be used for different diseases. We included two age groups, since there are many diseases that can be described using two age groups. This situation happens due to different conditions in each age group that would be unrealistic to average them to use only one age group. For instance, critical infections are usually presented in children, but not in adults. Thus, the infection outcomes are different for these two age groups. 

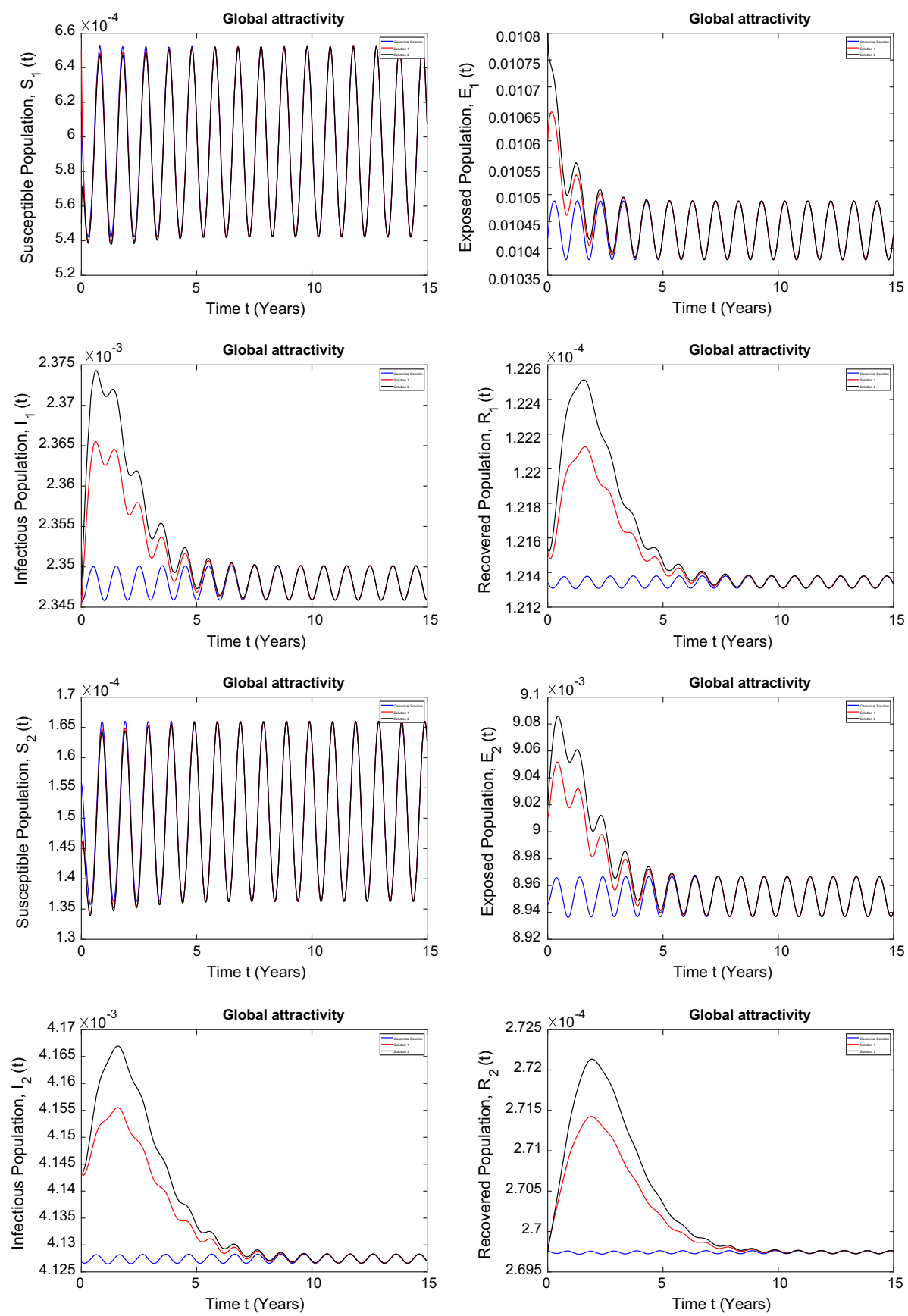

Fig. 1 Globally attractive behaviors of numerical solutions of system (4), using parameter values related to RSV in Australia. The canonical solution (blue) and two different solutions (red and black) corresponding to two initial conditions (color figure online) 
We presented numerical simulations to illustrate and corroborate the theoretical results obtained in this research work. In particular, we used as an example for the generalized force of infection the following form $f\left(I_{1}(t), I_{2}(t)\right)=\beta(t)\left(I_{1}(t)+I_{2}(t)\right)$, where $\beta(t)$ is a continuous $T$-periodic function. In regard to specific parameter values, we used reported RSV data from Australia, and performed numerical simulations where it can be seen the periodic behavior of the solution. Future directions of research include the introduction of noise in the harmonic seasonal forcing function to take into account uncertainty. In addition, modeling control policies such as treatment or vaccines are interesting topics and much more under climate variability. This type of studies can provide efficient public policies to reduce the prevalence of different diseases around the globe.

Acknowledgements The authors are grateful to the reviewers for their careful reading of this manuscript and their useful comments to improve the content of this paper. The first author gratefully acknowledges the partial funding from Universidad de Córdoba, Colombia, for this research.

\section{References}

Arenas AJ, González G, Jódar L (2008) Existence of periodic solutions in a model of respiratory syncytial virus RSV. J Math Anal Appl 344(2):969-980

Arenas AJ, González-Parra G, Moraño JA (2009) Stochastic modeling of the transmission of respiratory syncytial virus RSV in the region of Valencia, Spain. Biosystems 96(3):206-212

Ávila-Vales E, Rivero-Esquivel E, García-Almeida GE (2017) Global dynamics of a periodic SEIRS model with general incidence rate. Int J Differ Equ 2017:1-14

Azuma K, Kagi N, Kim H, Hayashi M (2020) Impact of climate and ambient air pollution on the epidemic growth during COVID-19 outbreak in Japan. Environ Res 190:110042

Bashir MF, Ma B, Komal B, Bashir MA, Tan D, Bashir M., et al. (2020) Correlation between climate indicators and COVID-19 pandemic in New York, USA. Science of The Total Environment p. 138835

Bezruchko BP, Smirnov DA (2000) Constructing nonautonomous differential equations from experimental time series. Phys Rev E 63(1):016207

Blackwood JC, Childs LM (2018) An introduction to compartmental modeling for the budding infectious disease modeler. Lett Biomath 5(1):195-221

Brauer F (2017) Mathematical epidemiology: past, present, and future. Infect Dis Model 2(2):113-127

Calatayud J, Jornet M (2020) Mathematical modeling of adulthood obesity epidemic in Spain using deterministic, frequentist and Bayesian approaches. Chaos Solitons Fractals 140:110179

Capasso V, Serio G (1978) A generalization of the Kermack-Mckendrick deterministic epidemic model. Math Biosci 42(1-2):43-61

Caraballo T, Han X (2017) Applied nonautonomous and random dynamical systems: applied dynamical systems. Springer

Chen F (2005) Periodicity in a ratio-dependent predator-prey system with stage structure for predator. J Appl Math 2005(2):153-169

Codeço CT (2001) Endemic and epidemic dynamics of cholera: the role of the aquatic reservoir. BMC Infect Dis $1(1): 1$

Coleman BD, Hsieh YH, Knowles GP (1979) On the optimal choice of $r$ for a population in a periodic environment. Math Biosci 46(1-2):71-85

Corberán-Vallet A, Santonja FJ, Jornet-Sanz M, Villanueva RJ (2018) Modeling chickenpox dynamics with a discrete time bayesian stochastic compartmental model. Complexity 2018

Derrick W, Van den Driessche P (1993) A disease transmission model in a nonconstant population. J Math Biol 31(5):495-512

Ding X, Zhao G (2012) Periodic solutions for a semi-ratio-dependent predator-prey system with delays on time scales. Discrete Dyn Nat Soc 2012:15

Donaldson GC (2006) Climate change and the end of the respiratory syncytial virus season. Clin Infect Dis 42(5):677-679

Fagan WF, Bewick S, Cantrell S, Cosner C, Varassin IG, Inouye DW (2014) Phenologically explicit models for studying plant-pollinator interactions under climate change. Theor Ecol 7(3):289-297 
Ferrero F, Torres F, Abrutzky R, Ossorio MF, Marcos A, Ferrario C, Rial MJ (2016) Seasonality of respiratory syncytial virus in Buenos Aires. Relationship with global climate change. Arch Argent Pediatr 114(1):525

Gaines RE, Mawhin JL (2006) Coincidence degree and nonlinear differential equations, vol 568. Springer, Berlin

Gao Dp, Huang Nj, Kang SM, Zhang C (2018) Global stability analysis of an SVEIR epidemic model with general incidence rate. Bound Value Probl 2018(1):42

Garrett Birkhoff GCR (1989) Ordinary differential equations, 4th edn. Wiley, New York

Gölgeli M, Atay FM (2020) Analysis of an epidemic model for transmitted diseases in a group of adults and an extension to two age classes. Hacettepe J Math Stat 49(3):921-934

González-Parra G, Arenas AJ, Jódar L (2009) Piecewise finite series solutions of seasonal diseases models using multistage adomian method. Commun Nonlinear Sci Numer Simul 14(11):3967-3977

Gopalsamy K (2013) Stability and oscillations in delay differential equations of population dynamics, vol 74. Springer

Guerrero-Flores S, Osuna O, Vargas-DeLeon C (2019) Periodic solutions for seasonal SIQRS models with nonlinear infection terms. Electron J Differ Equ 2019(92):1-13

Guo H, Chen X (2011) Existence and global attractivity of positive periodic solution for a Volterra model with mutual interference and Beddington-Deangelis functional response. Appl Math Comput 217(12):58305837

Hethcote HW, van den Driessche P (1991) Some epidemiological models with nonlinear incidence. J Math Biol 29:271-287

Hogan AB, Glass K, Moore HC, Anderssen RS (2016a) Age structures in mathematical models for infectious diseases, with a case study of respiratory syncytial virus. In: Anderssen R, et al (eds) Applications + practical conceptualization + mathematics = fruitful innovation. Mathematics for industry, vol 11 . Springer, Tokyo. https://doi.org/10.1007/978-4-431-55342-7_9

Hogan AB, Glass K, Moore HC, Anderssen RS (2016b) Exploring the dynamics of respiratory syncytial virus RSV transmission in children. Theor Popul Biol 110:78-85

Hogan AB, Campbell PT, Blyth CC, Lim FJ, Fathima P, Davis S, Moore HC, Glass K (2017) Potential impact of a maternal vaccine for RSV: a mathematical modelling study. Vaccine 35(45):6172-6179

Hurtado LA, Cáceres L, Chaves LF, Calzada JE (2014) When climate change couples social neglect: malaria dynamics in Panamá. Emerg Microbes Infect 3(1):1-11

Jia J, Zhang H (2014) Existence and global attractivity of periodic solutions for chemostat model with delayed nutrients recycling. Differ Equ Appl 6:275-286

Jódar L, Villanueva RJ, Arenas A (2008) Modeling the spread of seasonal epidemiological diseases: theory and applications. Math Comput Model 48(3):548-557

Jornet-Sanz M, Corberán-Vallet A, Santonja F, Villanueva R (2017) A Bayesian stochastic SIRS model with a vaccination strategy for the analysis of respiratory syncytial virus. SORT Stat Oper Res Trans 41(1):159_ 176

Korobeinikov A (2009) Global properties of SIR and SEIR epidemic models with multiple parallel infectious stages. Bull Math Biol 71:75-83

Li B, Xiong X (2010) Existence and global attractivity of periodic solution for a discrete prey-predator model with sex structure. Nonlinear Anal Real World Appl 11(3):1986-2000

Li Y, Qin J (2018) Existence and global exponential stability of periodic solutions for quaternion-valued cellular neural networks with time-varying delays. Neurocomputing 292:91-103

Li Y, Qin J, Li B (2019) Existence and global exponential stability of anti-periodic solutions for delayed quaternion-valued cellular neural networks with impulsive effects. Math Methods Appl Sci 42(1):5-23

Li Y, Teng Z, Ruan S, Li M, Feng X (2017) A mathematical model for the seasonal transmission of schistosomiasis in the lake and marshland regions of China. Math Biosci Eng 14:1279

De Luca G, Van Kerckhove K, Coletti P, Poletto C, Bossuyt N, Hens N, Colizza V (2018) The impact of regular school closure on seasonal influenza epidemics: a data-driven spatial transmission model for Belgium. BMC Infect Diseases 18(1):1-16

Malki Z, Atlam ES, Hassanien AE, Dagnew G, Elhosseini MA, Gad I (2020) Association between weather data and COVID-19 pandemic predicting mortality rate: machine learning approaches. Chaos Solitons Fractals 138:110137

Mateus JP, Silva CM (2017) Existence of periodic solutions of a periodic SEIRS model with general incidence. Nonlinear Anal Real World Appl 34:379-402

Mawhin J (2005) Periodic solutions in the golden sixties: the birth of a continuation theorem. In: Ferrera J, López-Gómez J, del Portal FR (eds) 10 mathematical essays on approximation in analysis and topology. Elsevier Science, Amsterdam, pp 199-214 
Mitchell C, Kribs C (2019) Invasion reproductive numbers for periodic epidemic models. Infect Dis Model 4:124-141

Noyola D, Mandeville P (2008) Effect of climatological factors on respiratory syncytial virus epidemics. Epidemiol Infect 136(10):1328-1332

Okuneye K, Abdelrazec A, Gumel AB (2018) Mathematical analysis of a weather-driven model for the population ecology of mosquitoes. Math Biosci Eng 15(1):57-93

Paiva TM, Ishida MA, Benega MA, Constantino CR, Silva DB, Santos KC, Oliveira MI, Barbosa HA, Carvalhanas TR, Schuck-Paim C et al (2012) Shift in the timing of respiratory syncytial virus circulation in a subtropical megalopolis: Implications for immunoprophylaxis. J Med Virol 84(11):1825-1830

Ponciano JM, Capistran MA (2011) First principles modeling of nonlinear incidence rates in seasonal epidemics. PLoS Comput Biol 7:1-14

Posny D, Wang J (2014) Modelling cholera in periodic environments. J Biol Dyn 8(1):1-19

Ren X, Zhang T (2017) Global analysis of an SEIR epidemic model with a ratio-dependent nonlinear incidence rate. J Appl Math Phys 5:2311-2319

Rogovchenko SP, Rogovchenko YV (2009) Effect of periodic environmental fluctuations on the Pearl-Verhulst model. Chaos Solitons Fractals 39(3):1169-1181

Rosa S, Torres DF (2018) Optimal control of a fractional order epidemic model with application to human respiratory syncytial virus infection. Chaos Solitons Fractals 117:142-149

Rui X, Lan-sun C, Fei-long H (2004) Periodic solutions of a delayed predator-prey model with stage structure for prey. Acta Math Appl Sin Engl Ser 20(2):323-332

Safi MA, Garba SM (2012) Global stability analysis of SEIR model with holling type II incidence function. Comput Math Methods Med 2012:1-8

Samanta S, Alquran M, Chattopadhyay J (2015) Existence and global stability of positive periodic solution of tri-trophic food chain with middle predator migratory in nature. Appl Math Model 39(15):4285-4299

Sekerci Y, Petrovskii S (2015) Mathematical modelling of plankton-oxygen dynamics under the climate change. Bull Math Biol 77(12):2325-2353

Sengupta S, Das P (2019) Dynamics of two-prey one-predator non-autonomous type-III stochastic model with effect of climate change and harvesting. Nonlinear Dyn 97(4):2777-2798

Shobugawa Y, Takeuchi T, Hibino A, Hassan MR, Yagami R, Kondo H, Odagiri T, Saito R (2017) Occurrence of human respiratory syncytial virus in summer in Japan. Epidemiol Infect 145(2):272-284

Traoré B, Sangaré B, Traoré S (2017) A mathematical model of malaria transmission with structured vector population and seasonality. J Appl Math 2017

Valle SYD, Hyman JM, Chitnis N (2013) Mathematical models of contact patterns between age groups for predicting the spread of infectious diseases. Math Biosci Eng 10:1475

Vega YL, Ramirez OV, Herrera BA (2017) Impact of climatic variability in the respiratory syncytial virus pattern in childrenunder 5 years-old using the Bulto climatic index in Cuba. Int J Virol Infect Dis 2:1419

Wang L (2015) Existence of periodic solutions of seasonally forced SIR models with impulse vaccination. Taiwan J Math 19(6):1713-1729

Weber A, Weber M, Milligan P (2001) Modeling epidemics caused by respiratory syncytial virus RSV. Math Biosci 172:95-113

White L, Mandl J, Gomes M, Bodley-Tickell A, Cane P, Perez-Brena P, Aguilar J, Siqueira M, Portes S, Straliotto S, Waris M, Nokes D, Medley G (2007) Understanding the transmission dynamics of respiratory syncytial virus using multiple time series and nested models. Math Biosci 209:222-239

White L, Waris M, Cane P, Nokes D, Medley G (2005) The transmission dynamics of groups A and B human respiratory syncytial virus hRSV in England and Wales and Finland: seasonality and cross-protection. Epidemiol Infect 133:279-289

Yan Y, Sugie J (2020) Global asymptotic stability of a unique positive periodic solution for a discrete hematopoiesis model with unimodal production functions. Monatshefte Math 191(2):325-348

Zhang T, Liu J, Teng Z (2012) Existence of positive periodic solutions of an SEIR model with periodic coefficients. Appl Math 57(6):601-616

Zhu Y, Wang K (2011) Existence and global attractivity of positive periodic solutions for a predator-prey model with modified Leslie-Gower Holling-type II schemes. J Math Anal Appl 384(2):400-408

$\mathrm{Zu}$ J, Wang L (2015) Periodic solutions for a seasonally forced SIR model with impact of media coverage. Adv Differ Equ 136:1-10

Publisher's Note Springer Nature remains neutral with regard to jurisdictional claims in published maps and institutional affiliations. 